\title{
In-Depth Search Focused on Furans, Lactones, Volatile Phenols, and Acetals As Potential Age Markers of Madeira Wines by Comprehensive Two-Dimensional Gas Chromatography with Time-of-Flight Mass Spectrometry Combined with Solid Phase Microextraction
}

\author{
Rosa Perestrelo, ${ }^{+, \neq}$António S. Barros, ${ }^{\dagger}$ José S. Câmara, ${ }^{\ddagger}, \S$ and Sílvia M. Rocha ${ }^{*,+}$ \\ ${ }^{\dagger}$ QOPNA, Departamento de Química, Universidade de Aveiro, 3810-193 Aveiro, Portugal \\ ${ }^{\ddagger}$ Centro de Química da Madeira, Campus Universitário da Penteada, 9000-390 Funchal, Portugal \\ ${ }^{\S}$ Centro de Ciências Exactas e de Engenharia da Universidade da Madeira, Campus Universitário da Penteada, 9000-390 Funchal, \\ Portugal
}

\begin{abstract}
The establishment of potential age markers of Madeira wine is of paramount significance as it may contribute to detect frauds and to ensure the authenticity of wine. Considering the chemical groups of furans, lactones, volatile phenols, and acetals, 103 volatile compounds were tentatively identified; among these, 71 have been reported for the first time in Madeira wines. The chemical groups that could be used as potential age markers were predominantly acetals, namely, diethoxymethane, 1,1diethoxyethane, 1,1-diethoxy-2-methyl-propane, 1-(1-ethoxyethoxy)-pentane, trans-dioxane and 2-propyl-1,3-dioxolane, and from the other chemical groups, 5-methylfurfural and cis-oak-lactone, independently of the variety and the type of wine. GC $\times$ GCToFMS system offers a more useful approach to identify these compounds compared to previous studies using GC-qMS, due to the orthogonal systems, that reduce coelution, increase peak capacity and mass selectivity, contributing to the establishment of new potential Madeira wine age markers. Remarkable results were also obtained in terms of compound identification based on the organized structure of the peaks of structurally related compounds in the GC $\times$ GC peak apex plots. This information represents a valuable approach for future studies, as the ordered-structure principle can considerably help the establishment of the composition of samples. This new approach provides data that can be extended to determine age markers of other types of wines.
\end{abstract}

KEYWORDS: Age markers, Madeira wine, HS-SPME, GC × GC-ToFMS

\section{INTRODUCTION}

Madeira wine is a fortified Portuguese wine produced in Madeira Island over the last centuries and plays an important role in the economy of the Island. The peculiar characteristics of Madeira wines arise from the specific and singular winemaking process. The fermentation process is stopped by the addition of natural grape spirit in order to obtain an ethanol content of $18-$ $22 \%(\mathrm{v} / \mathrm{v})$. Some wines undergo aging in wood casks in cellars at temperatures up to $30{ }^{\circ} \mathrm{C}$, and humidity levels between 70 and $75 \%$, while the majority of wines are submitted to a baking process, i.e., the wine is placed in large coated vats, and the temperature is slowly increased at about $5^{\circ} \mathrm{C}$ per day and maintained at $45-$ $50{ }^{\circ} \mathrm{C}$ during at least 3 months. After this treatment, the wine is allowed to undergo a maturation process in oak casks for a minimum of 3 years. Finally, some Madeira wines were submitted to an aging process, from a minimum of 3 to 20 years or even longer. ${ }^{1,2}$ The aging process in oak casks is fundamental for the Madeira wine's unique sensorial properties. During this period, several reactions and migration of molecules from the oak to wine can occur, ${ }^{3,4}$ which depends on some parameters, such as grape variety, wine making procedure, and oak characteristics (geographical origin, species of oak, seasoning of the staves, toasting, and age of cask), ${ }^{5-9}$ among others.

The establishment of potential age markers is important to detect frauds and to ensure the authenticity of the wine. Furthermore, the economic value of Madeira wine is highly associated with its age. Some volatile compounds that belong to furans, lactones, volatile phenols, and acetals have been reported as potential aging markers in Madeira wines. ${ }^{4,10-12}$ Compounds such as, 2-furfural, 5-methylfurfural, 5-hydroxymethylfurfural, cisoak-lactone, trans-oak-lactone, eugenol, guaiacol, $m$-cresol, $o$ cresol, p-ethylphenol, maltol, vanillin, cis-dioxane, trans-dioxane, cis-dioxolane, and trans-dioxolane were considered. ${ }^{5,13-16}$ Furans (e.g., 2-furfural, 5-methylfurfural, and 5-hydroxymethyl-2-furfural) are formed by three pathways: pyrolysis of carbohydrates, dehydration of sugars through Maillard reaction, and caramelization, ${ }^{17-19}$ which occurs during winemaking and aging. As the levels of 2-furfural and 5-hydroxymethyl-2-furfural have a tendency to increase linearly during aging, they were considered as age markers. ${ }^{4,20}$ The lactones are important flavor compounds which are produced by cyclization of the corresponding hydroxycarboxylic acids. ${ }^{4,21}$ Oak lactones, such as cis- and trans-oaklactone, are already present in natural oak, and their content increased due to seasoning and toasting, ${ }^{14}$ and from an organoleptic point of view, they are the most important lactones extractable from oak casks. ${ }^{22}$ Volatile phenols, like ethyl and vinylphenols, were also extracted from oak; nevertheless, their microbiological yeast transformation (e.g., Brettanomyces and

Received: November 2, 2010

Accepted: February 1, 2011

Revised: January 10, 2011

Published: March 04, 2011 
Dekkara) from hydroxycinnamic acids of wine were reported as the main origin. ${ }^{23}$ Acetals are formed during fermentation; nevertheless, their content increases significantly during the oxidative conditions of aging process. The high acetaldehyde content in wine contributes to the acetalization reaction with glycerol, which is favored at higher $\mathrm{pH}$ values, leading to four heterocyclic acetal alcohol formation: cis- and trans-5-hydroxy-2methyl-1,3-dioxane (cis-dioxane and trans-dioxane), and cis- and trans-4-hydroxymethyl-2-methyl-1,3-dioxalane (cis-dioxolane and trans-dioxolane). Heterocyclic acetal alcohols were identified and reported as potential age markers of Madeira wine., ${ }^{4,10,11}$ Other acetals, such as 1,1-diethoxyethane and 2,4,5-trimethyldioxolane, were also detected in table wines. ${ }^{24}$

The Madeira wine volatile composition related to aging process has been studied using a one-dimensional chromatographic $\left({ }^{1} \mathrm{D}\right.$ GC) process, which revealed the complexity of this matrix., ${ }^{4,10-12}$ Although such a method often provides rewarding analytical results, in-depth analysis of the chromatograms frequently indicates that some peaks are the result of two or more coeluting compounds. Comprehensive two-dimensional gas chromatography $(\mathrm{GC} \times \mathrm{GC})$ was developed as a powerful separation method and emerged as an interesting alternative to analyze complex samples or analyze trace target analytes within a single analysis and overcoming the coelution problem. ${ }^{25}$ The method employs two orthogonal mechanisms and is based on the application of two GC columns coated with different stationary phases, a nonpolar and a polar one $(\mathrm{NP} / \mathrm{P})$, sequentially linked through a modulator. Thus, the separation is ruled by boiling point properties in the first dimension $\left({ }^{1} \mathrm{D}\right)$ and polarity in the second one $\left({ }^{2} \mathrm{D}\right){ }^{26,27}$ Therefore, two-dimensional gas chromatography $(\mathrm{GC} \times \mathrm{GC})$ offers faster running times, increased peak capacity, improved resolution and enhanced mass selectivity, good calibration linearity, and more sensitivity, and the limits of detection are improved due to the focusing of the peak in the modulator when compared to that in the one-dimensional $\mathrm{GC}^{28-30}$

In order to obtain a deeper characterization of the chemical groups potentially related with Madeira wine aging, namely, furans, lactones, volatile phenols, and acetals, the comprehensive two-dimensional gas chromatography with time-of-flight mass spectrometry $(\mathrm{GC} \times \mathrm{GC}-\mathrm{ToFMS})$ combined with headspace solid-phase microextraction (HS-SPME) was used in the present research. This methodology was applied to Madeira wines from different varieties (Malvasia, Bual, Sercial, Verdelho, and Tinta Negra), types (sweet, medium sweet, dry, and medium dry), and ages (Vintage and blended wines). Finally, principal component analysis (PCA) was applied in order to establish potential age markers, which allow one to distinguish the different types of Madeira wines based on their age and even blends (average age).

\section{MATERIAL AND METHODS}

Samples. Twenty-three monovarietal Madeira wines from five Vitis vinifera L. grape varieties (one red, Tinta Negra, and four white, named as noble varieties of Madeira wine, Malvasia, Bual, Sercial, and Verdelho), aged from 3 to 20 years old $(\mathrm{Y})$ and matured in oak casks, were used in this study. Tinta Negra is the main grape variety harvested in Madeira Island (Portugal) representing more than $80 \%$ of the vineyards. According to the age, the wines under study correspond to Vintage (a specific year of aged in casks, 17, 18, 19, and 20 years) and blended (B, an average aging period of 3, 5, 10, or 15 years) wines. Four types of wine were used: sweet (Malvasia, Tinta Negra), medium sweet (Bual, Tinta Negra), dry (Sercial, Tinta Negra), and medium dry (Verdelho, Tinta Negra), and were aged in American oak casks (submitted to a lighter toasting). The ethanol content of the Madeira wines under study ranged from 18 to $19 \%$ (v/v). The samples were kindly provided by Madeira Wine Company, Madeira Island.

Reagents and Standards. Sodium chloride (99.5\%, foodstuff grade) was purchased from Sigma Aldrich (Madrid, Spain), and ultra pure water was obtained from a Milli-Q system from Millipore (Milford, MA, USA). The retention index probes ( $n$-alkanes series of $C_{8}$ to $C_{20}$ straight-chain alkanes, concentration $40 \mathrm{mg} / \mathrm{L}$ in $n$-hexane) were supplied from Fluka (Buchs, Switzerland).

HS-SPME Methodology. The HS-SPME experimental parameters were previously established. ${ }^{2}$ The SPME holder for manual sampling and fiber were purchased from Supelco (Aldrich, Bellefonte, PA, USA). The SPME device included a fused silica fiber coating partially cross-linked with $50 / 30 \mu \mathrm{m}$ divinylbenzene-carboxen-poly(dimethylsiloxane). Prior to use, the SPME fiber was conditioned at $270{ }^{\circ} \mathrm{C}$ for $60 \mathrm{~min}$ in the GC injector, according to the manufacturer's recommendations. Then, the fiber was daily conditioned for $10 \mathrm{~min}$ at $250{ }^{\circ} \mathrm{C}$.

For the HS-SPME assay, aliquots of $1 \mathrm{~mL}$ of the sample were placed into a $5 \mathrm{~mL}$ glass vial. After the addition of $0.5 \mathrm{~g}$ of $\mathrm{NaCl}$ and stirring $(0.5 \times 0.1 \mathrm{~mm}$ bar $)$ at $400 \mathrm{rpm}$, the vial was capped with a PTFE septum and an aluminum cap (Chromacol, Hertfordshire, UK). The vial was placed in a thermostatted bath adjusted to $60.0 \pm 0.1{ }^{\circ} \mathrm{C}$ for $5 \mathrm{~min}$, and then the SPME fiber was inserted in the headspace for $20 \mathrm{~min}$. Each sample was analyzed in triplicate. Blanks, corresponding to the analysis of the coating fiber not submitted to any extraction procedure, were run between sets of three analyses.

GC $\times$ GC-ToFMS Analysis. The GC $\times$ GC-ToFMS methodology was based on a previous study. ${ }^{2}$ After the extraction/concentration step, the SPME coating fiber was manually introduced into the GC $\times$ GC-ToFMS injection port at $250^{\circ} \mathrm{C}$ and kept for $3 \mathrm{~min}$ for desorption. The injection port was lined with a $0.75 \mathrm{~mm}$ I.D. splitless glass linear. Splitless injections were used (30 s). LECO Pegasus 4D (LECO, St. Joseph, MI, USA) GC $\times$ GC-ToFMS system consisted of an Agilent GC 7890A gas chromatograph, with a dual stage jet cryogenic modulator (licensed from Zoex) and a secondary oven. The detector was a highspeed ToF mass spectrometer. An HP-5 column ( $30 \mathrm{~m} \times 0.32 \mathrm{~mm}$ I.D., $0.25 \mu \mathrm{m}$ film thickness, J\&W Scientific Inc., Folsom, CA, USA) was used as first-dimension column, and a DB-FFAP $(0.79 \mathrm{~m} \times 0.25 \mathrm{~mm}$ I.D., $0.25 \mu \mathrm{m}$ film thickness, J\&W Scientific Inc., Folsom, CA, USA) was used as a second-dimension column. The carrier gas was helium at a constant flow rate of $2.50 \mathrm{~mL} / \mathrm{min}$. The primary oven temperature was programmed from $40(1 \mathrm{~min})$ to $230{ }^{\circ} \mathrm{C}(2 \mathrm{~min})$ at $10{ }^{\circ} \mathrm{C} / \mathrm{min}$. The secondary oven temperature was programmed from $70(1 \mathrm{~min})$ to $250{ }^{\circ} \mathrm{C}(3 \mathrm{~min})$ at $10^{\circ} \mathrm{C} / \mathrm{min}$. The MS transfer line temperature was $250{ }^{\circ} \mathrm{C}$, and the MS source temperature was $250^{\circ} \mathrm{C}$. The modulation time was $6 \mathrm{~s}$; the modulator temperature was kept at $20^{\circ} \mathrm{C}$ offset (above primary oven). A $6 \mathrm{~s}$ modulation time with a $30^{\circ} \mathrm{C}$ secondary oven temperature offset was chosen to be a suitable compromise as it maintained the $1 \mathrm{D}$ separation, maximized the $2 \mathrm{D}$ resolution, and avoided the wrap-around effect (the elution time of a pulsed solute exceeds the modulation period) for compounds that were late to elute from the $2 \mathrm{D}$. Ideally, all peaks must be detected before the subsequent reinjection, and hence, ${ }^{2} t_{\mathrm{R}}$ must be equal or less than the modulation period. $^{31,32}$ The ToFMS was operated at a spectrum storage rate of 125 spectra/s. The mass spectrometer was operated in the EI mode at $70 \mathrm{eV}$ using a range of $m / z 33-350$, and the voltage was $-1695 \mathrm{~V}$. Total ion chromatograms (TIC) were processed using the automated data processing software ChromaTOF (LECO) at a signal-to-noise threshold of 10 . Contour plots were used to evaluate the separation general quality and for manual peak identification; a signal-to-noise threshold of 50 was used. Two commercial databases (Wiley 275 and US National Institute of Science and Technology (NIST) V. 2.0, Mainlib and Replib) were used. A mass spectral match factor, the majority ( $86 \%)$ of the tentatively 


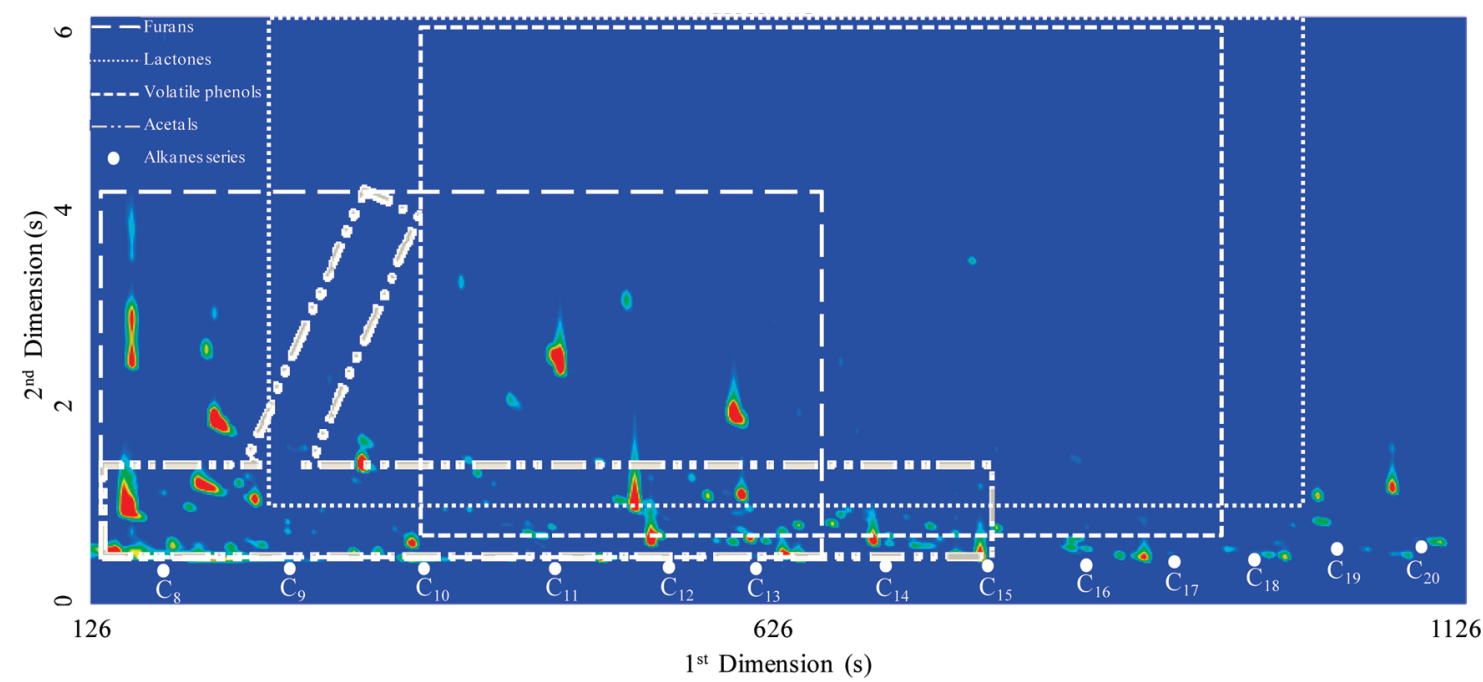

Figure 1. GC $\times$ GC total ion current chromatogram contour plot obtained from a sweet Madeira wine (Tinta Negra, 5 years); the chromatographic spaces corresponding to furans, lactones, volatile phenols, and acetals were highlighted. The $n$-alkanes series $\left(\mathrm{C}_{6}-\mathrm{C}_{20}\right)$ was superimposed on the contour plot.

identified compounds showed similarity matches $>850$, was set to decide whether a peak was correctly identified or not. Furthermore, a manual inspection of the mass spectra was done, combined with the use of additional data, such as the retention index (RI) value, which was determined according to the Van den Dool and Kratz RI equation. ${ }^{33}$ For the determination of the RI, a $\mathrm{C}_{8}-\mathrm{C}_{20} n$-alkanes series was used, and as some volatile compounds were eluted before $\mathrm{C}_{8}$, the solvent $n$-hexane was used as the $\mathrm{C}_{6}$ standard. The $\mathrm{RI}$ values experimentally calculated were compared, when available, with values reported in the literature for similar chromatographic columns that employ as the first dimension the column of this study. ${ }^{34-51}$ The GC $\times$ GC area data were used as an approach to estimate the relative content of each volatile component.

Data Processing. In an initial approach, a linear regression was performed between total GC peak area of the chemical groups (furans, lactones, volatile phenols, and acetals) under study and wine age in order to establish potential age markers for Malvasia and Bual wines, and the results were expressed as $r^{2}$ ( coefficient of determination). In a second step, PCA (principal component analysis) was applied to the autoscaled areas of the 103 volatile compounds tentatively identified by HS-SPME/ $\mathrm{GC} \times \mathrm{GC}-\mathrm{ToFMS}$ present in 23 monovarietal Madeira wines (from different varieties, types, and age) each with three replicates, using the $\mathrm{R}$ statistical software package. ${ }^{52}$ Autoscaling is a data pretreatment process that makes variables of different scales comparable. Each variable is autoscaled separately by subtracting its mean value and dividing by its standard deviation. The goal was to extract the main sources of variability and hence to help with the characterization of the data set. ${ }^{53}$

\section{RESULTS AND DISCUSSION}

Contour and Peak Apex Plot Analysis. Automated processing of HS-SPME/GC $\times$ GC-ToFMS data was used to tentatively identify all peaks in the GC $\times$ GC chromatogram contour plots with a signal-to-noise threshold $>50$. The contour plot of the total ion chromatogram (Figure 1) exhibited several hundreds of peaks; however, this study was only focused on furans, lactones, volatile phenols, and acetals. The peak finding routine based on the deconvolution method allowed us to detect 103 compounds from these four chemical groups, which were tentatively identified on the basis of the comparison of their mass spectra to a reference database (MS) and by comparison of the RIs calculated $\left(\mathrm{RI}_{\text {calc }}\right)$ with the values reported in the literature $\left(\mathrm{RI}_{\mathrm{lit}}\right)$ for the 5\% phenylpolysilphenylene-siloxane (or equivalent) column (Tables 1 and 2). A range between 1 and $30\left(\left|\mathrm{RI}_{\text {calc }}-\mathrm{RI}_{\text {lit }}\right|\right)$ was obtained for $\mathrm{RI}_{\text {cal }}$ compared to the $\mathrm{RI}_{\text {lit }}$ reported in the literature for one-dimensional GC with the 5\%-phenyl-methylpolysiloxane GC column or equivalent. This difference in RI is considered reasonable $(<5 \%)$ if one takes into account that $(i)$ the literature data is obtained from a large range of GC stationary phases (several commercial GC columns are composed of 5\% phenylpolysilphenylene-siloxane or equivalent stationary phases) and that (ii) the literature values were determined in a onedimensional chromatographic separation system, and the modulation causes some inaccuracy in the first dimension retention time. ${ }^{54}$ In the case of the volatile compounds with $\left.\left|\mathrm{RI}_{\text {calc }}-\mathrm{R}_{\text {lit }}\right|\right)$ values higher than 30 , the information related to the mass spectra $(\mathrm{m} / \mathrm{z})$ was included in Tables 1 and 2.

Figure 1 shows the GC $\times$ GC total ion current chromatogram contour plot obtained from a sweet Madeira wine (Tinta Negra, $5 \mathrm{Y})$; the chromatographic spaces corresponding to furans, lactones, volatile phenols, and acetals were highlighted. The $n$ alkanes series $\left(\mathrm{C}_{6}-\mathrm{C}_{20}\right)$ used for the calculation of experimental RIs are also superimposed on the contour plot. The components of each chemical group were dispersed through the contour plot according to their volatility $\left({ }^{\mathrm{P}} \mathrm{D}\right)$ and polarity $\left({ }^{2} \mathrm{D}\right)$, and it becomes difficult to establish the two-dimension chromatographic space $(\mathrm{GC} \times \mathrm{GC})$ specific for each chemical group. As the principle of the structured chromatogram is very important in the identification, especially for the compounds that are not commercially available, a strategy was implemented to find this principle. Thus, peak apex plots were constructed, in order to find the possible structured $2 \mathrm{D}$ chromatographic profile, combining ${ }^{1} t_{\mathrm{R}}$ and ${ }^{2} t_{\mathrm{R}}$ values, for each chemical group under study, as shown for furans (Figure 2), lactones (Figure 3), volatile phenols (Figure 4), and acetals (Figure 5). Peak apex plots indicate the position of the maximum modulated peak of GC $\times$ GC analysis, in the $2 \mathrm{D}$ chromatographic space. ${ }^{55}$ For all chemical groups, as expected, it was observed that the decrease in volatility (high ${ }^{1} t_{\mathrm{R}}$ ) is mainly related to the increase in the number of carbons.

The furans include several types of chemical structures; thus, they were organized in furan/alkyl furan, furanic aldehyde, furanic alcohol, benzofuran, furanic ester, and furanic acetal 


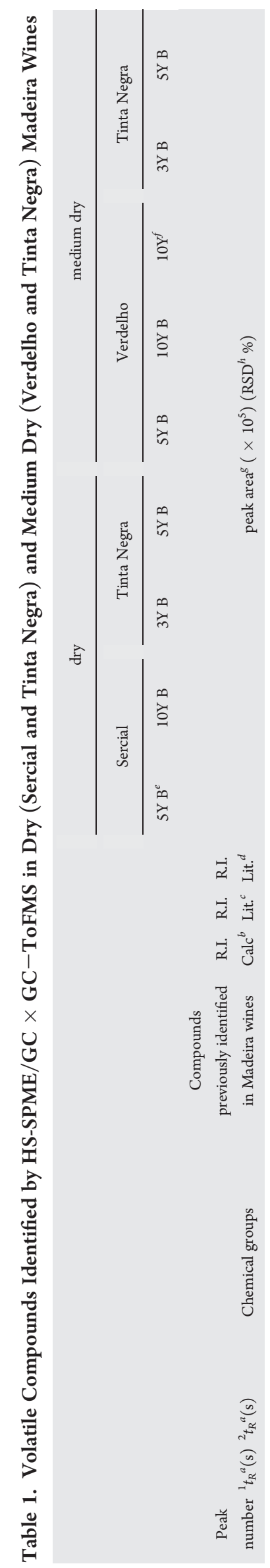

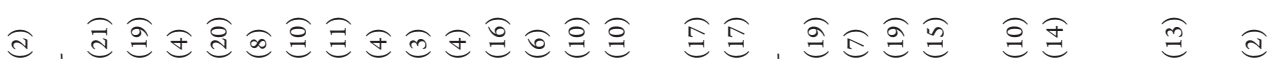

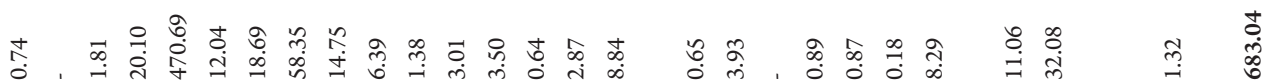

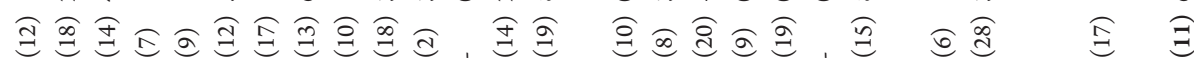

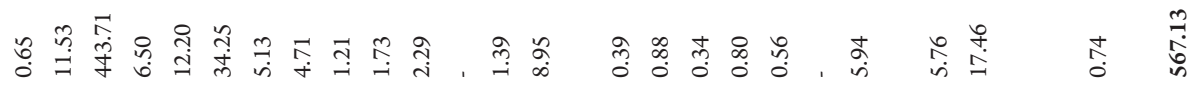

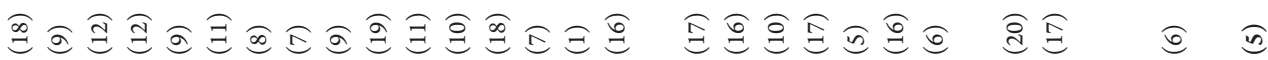

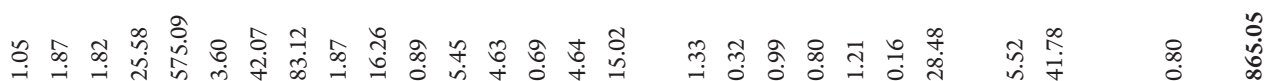

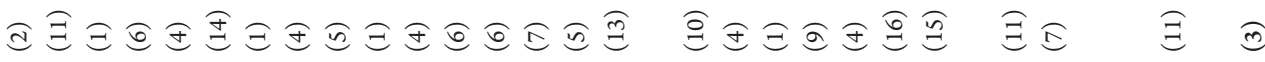

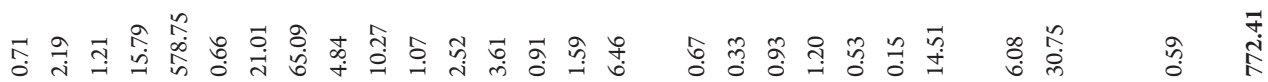

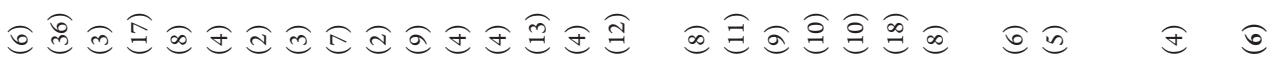

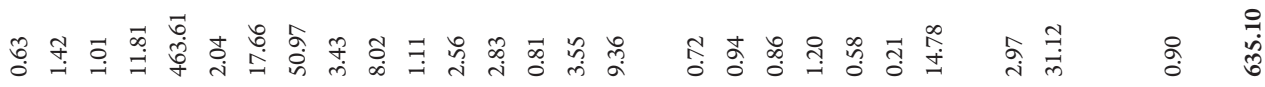

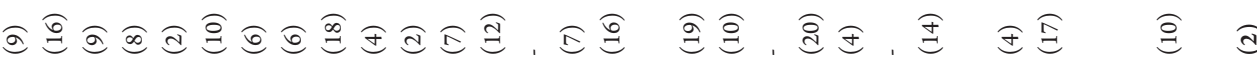

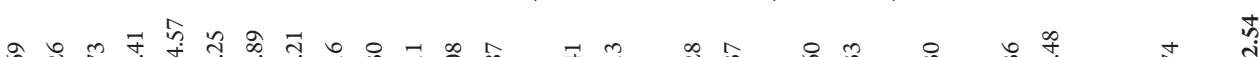

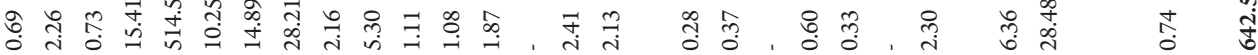

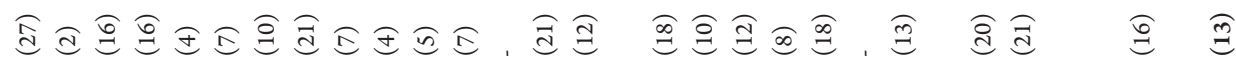

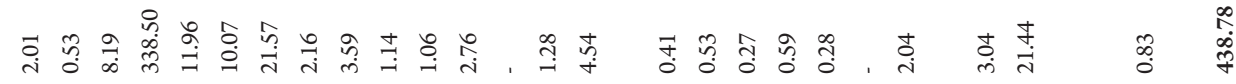

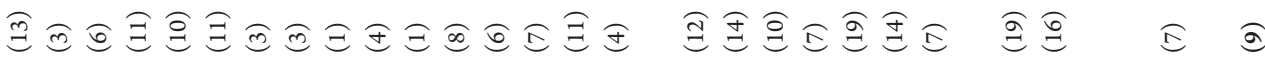

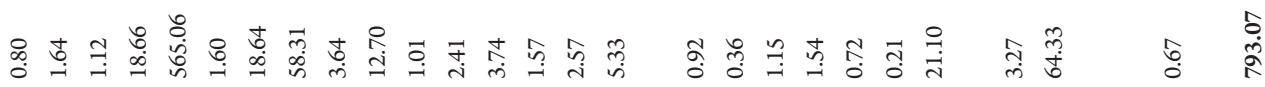

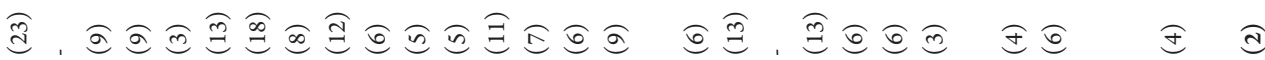

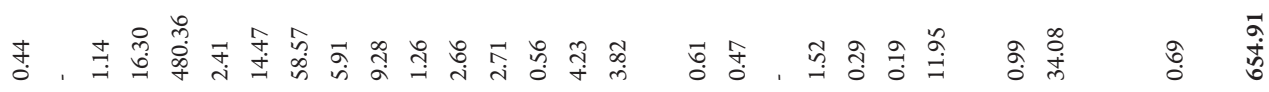

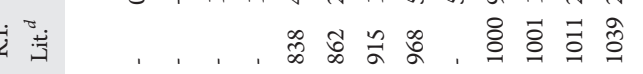

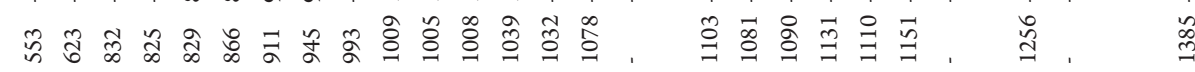

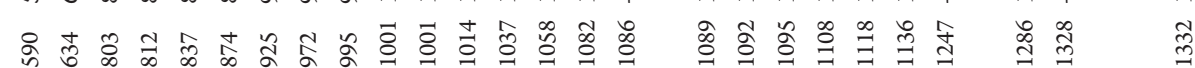

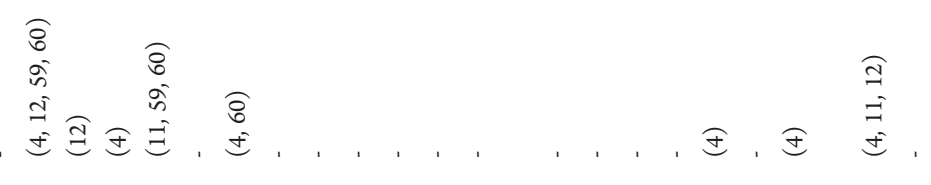

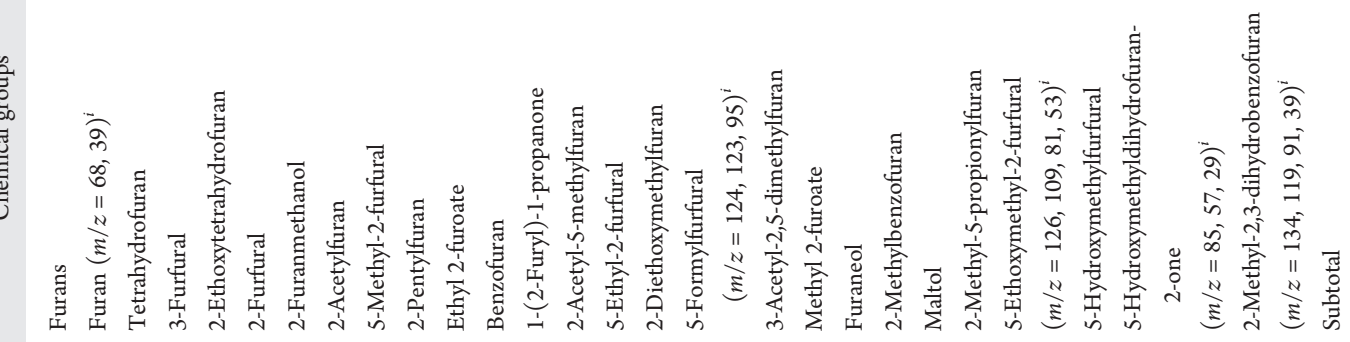

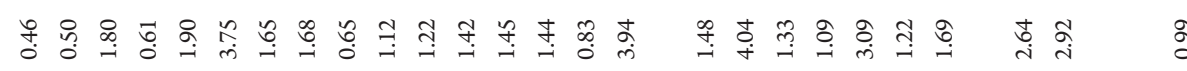

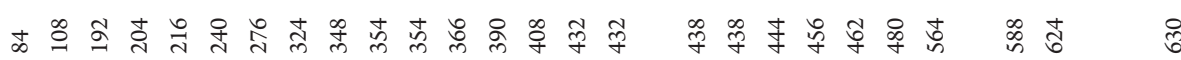
$-\lambda \infty a$ 글 


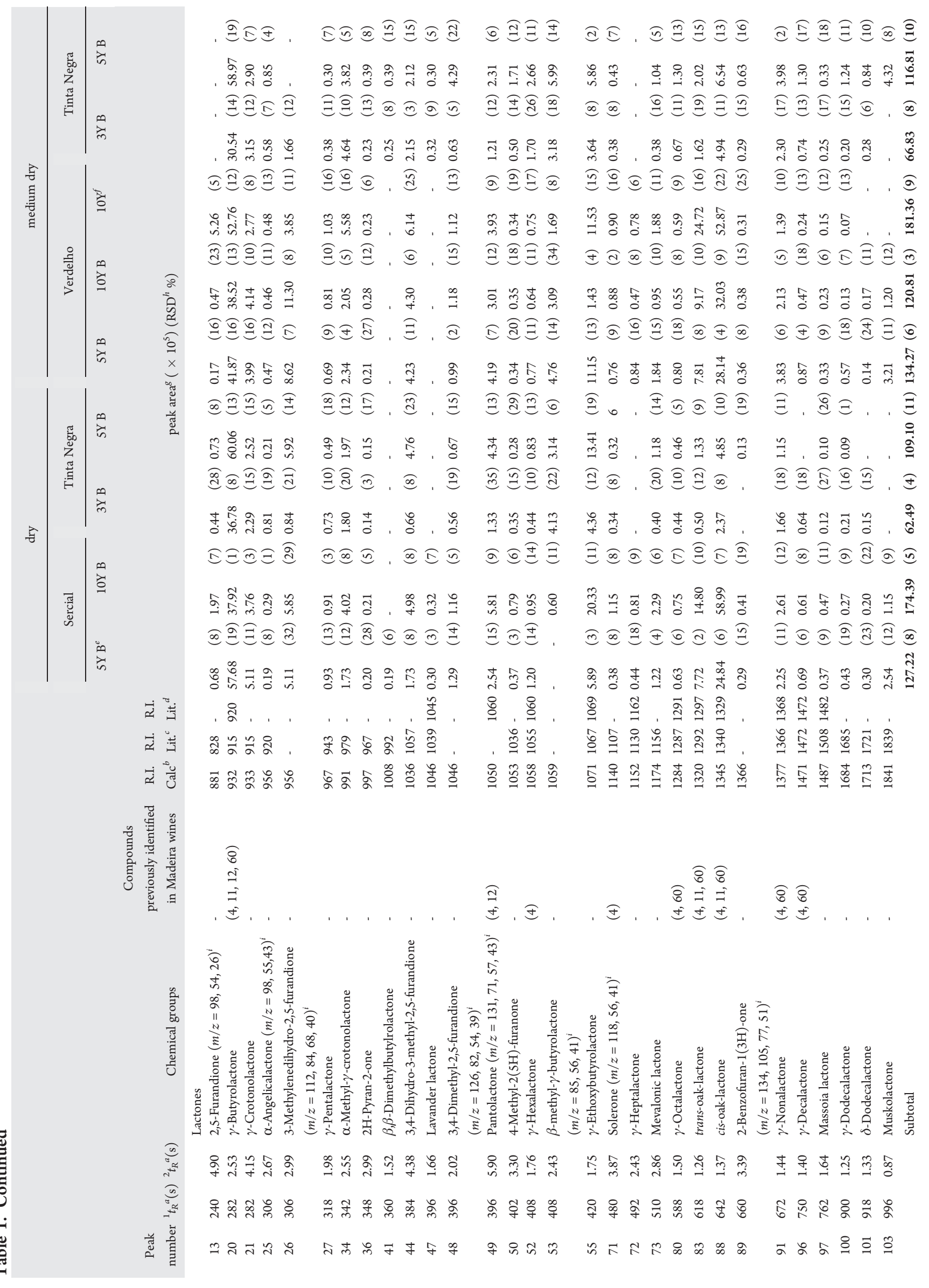




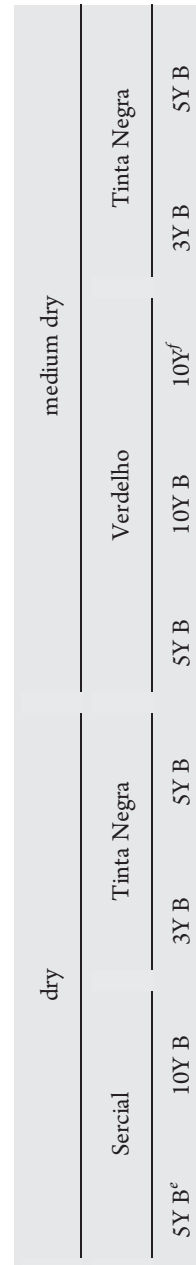

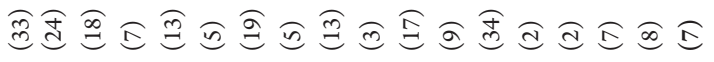

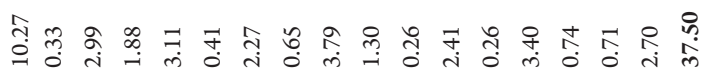

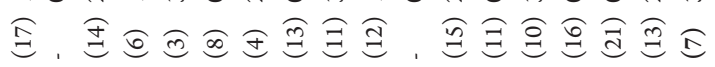

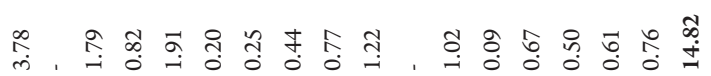

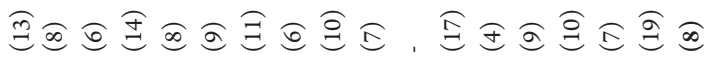

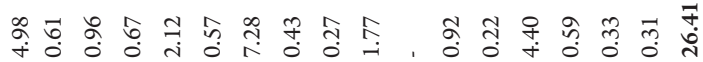

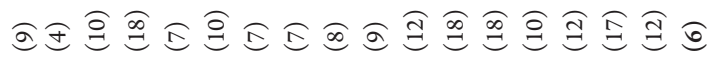

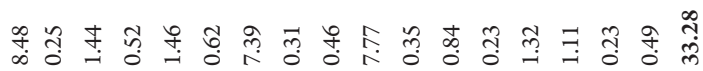

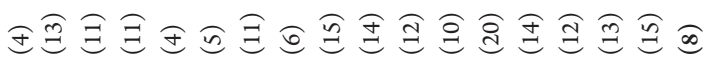

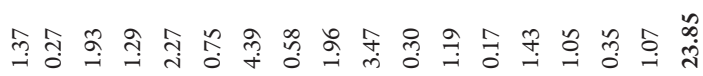

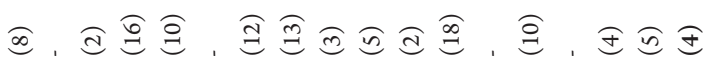

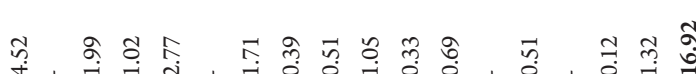

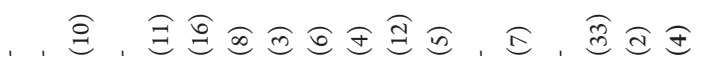

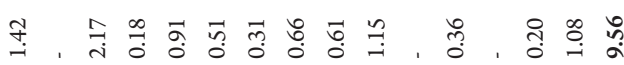

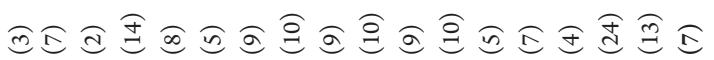

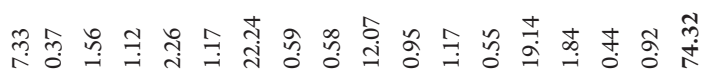

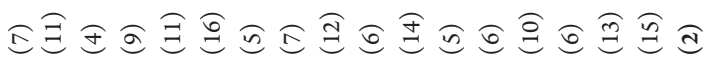

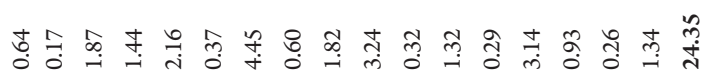

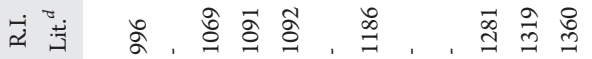

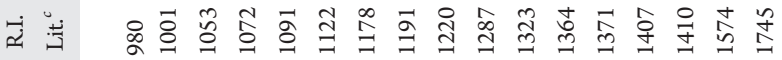

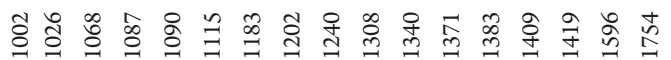

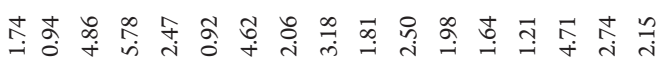

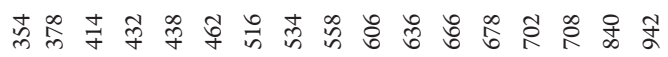

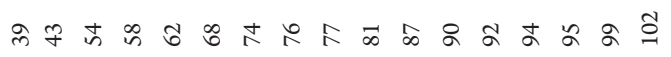

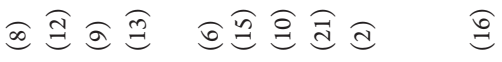

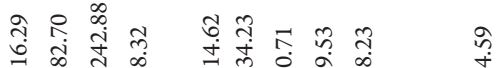

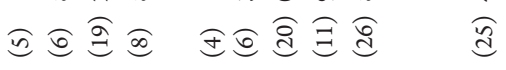

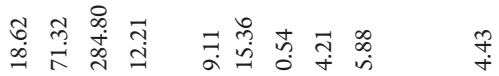

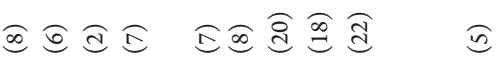

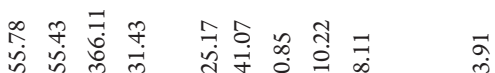

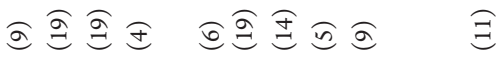

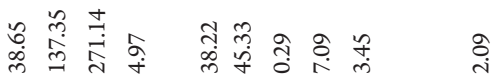

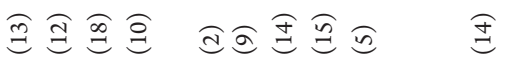

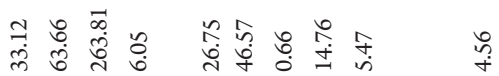

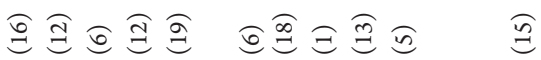

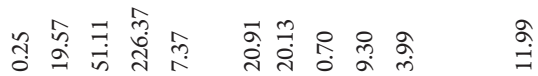

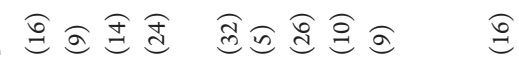

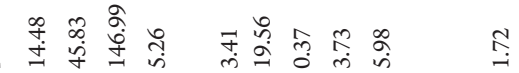

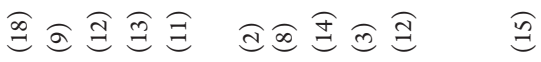

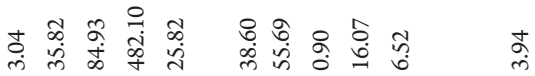

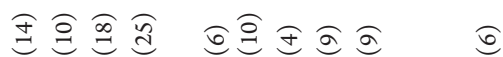

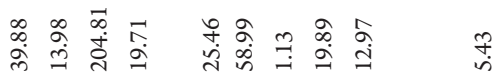

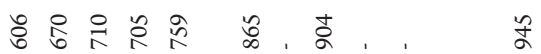

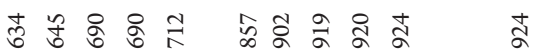

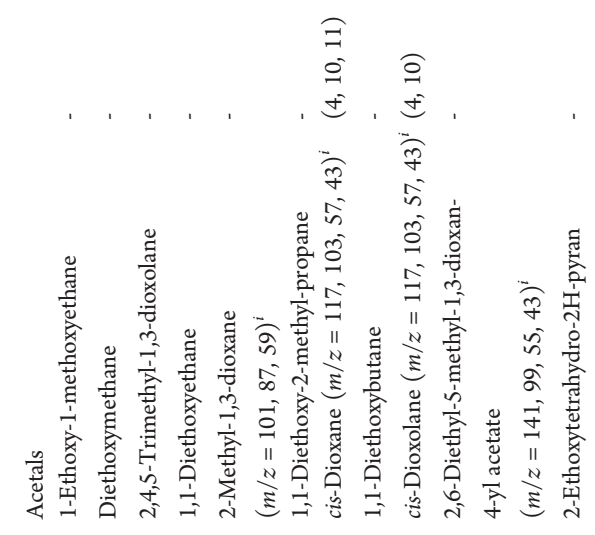

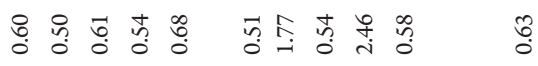

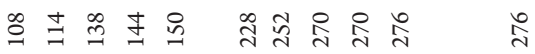




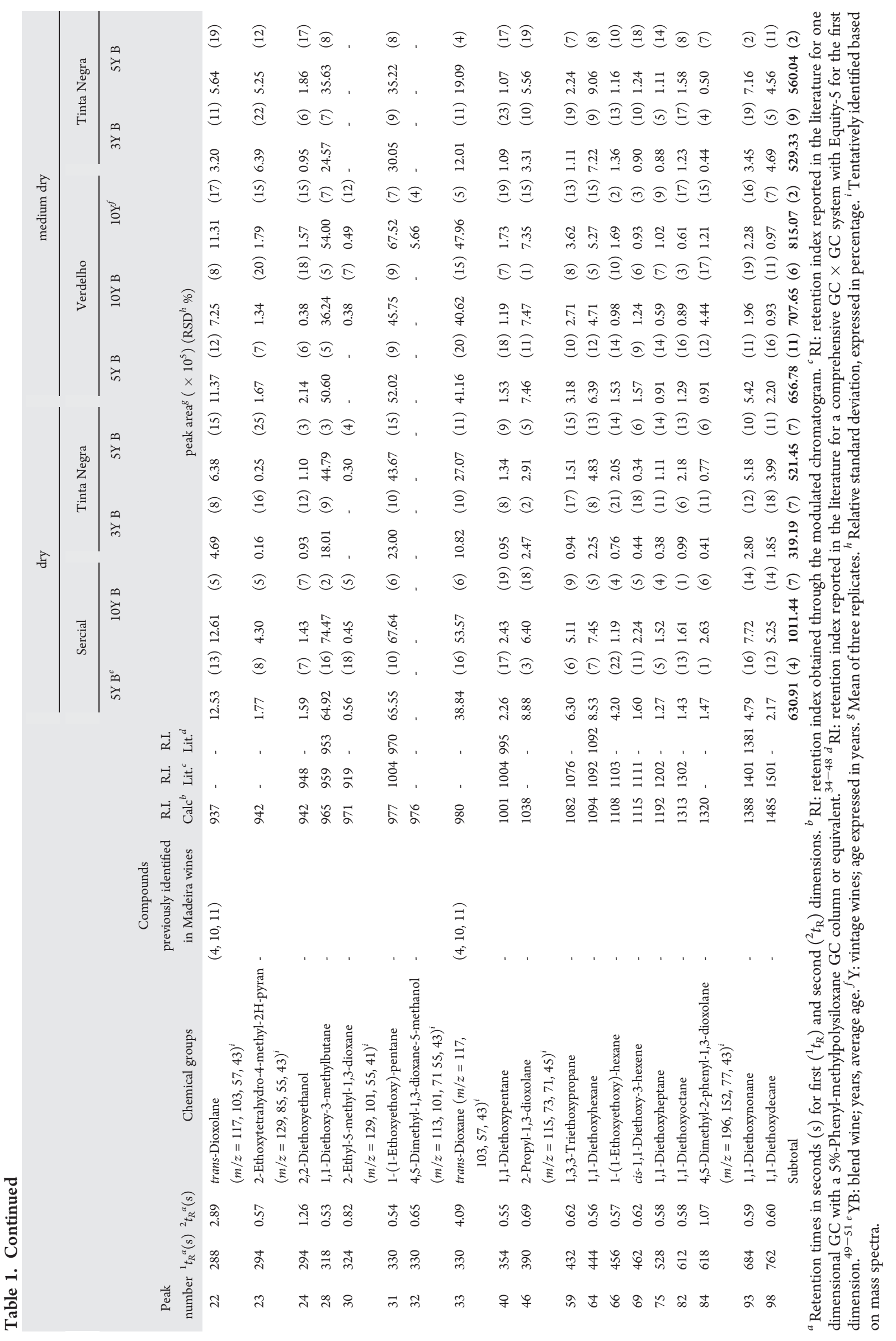




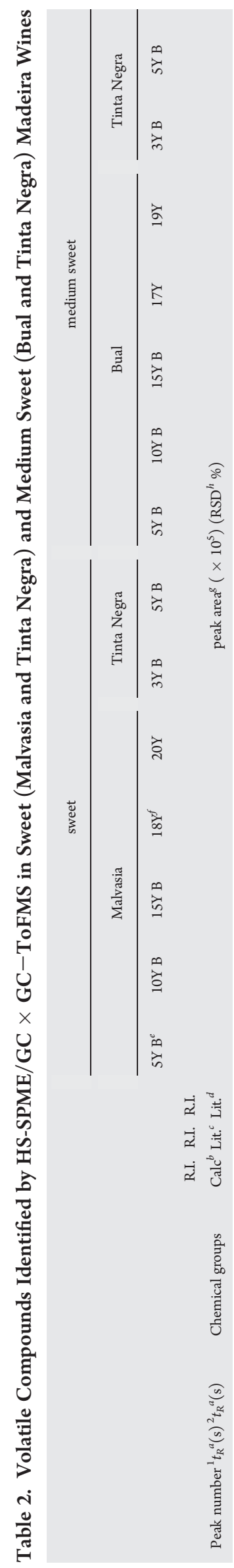

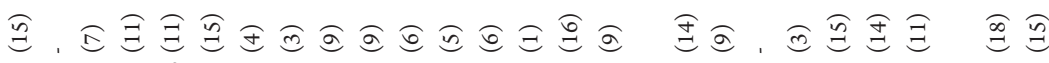

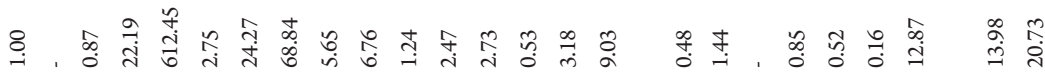

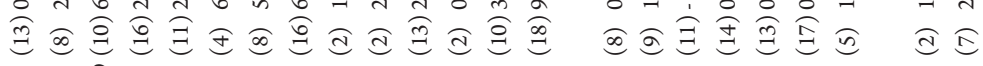

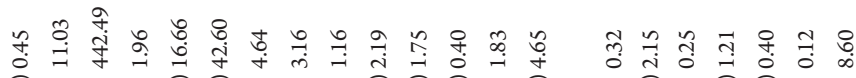

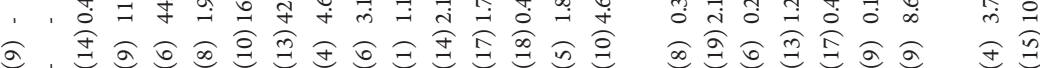

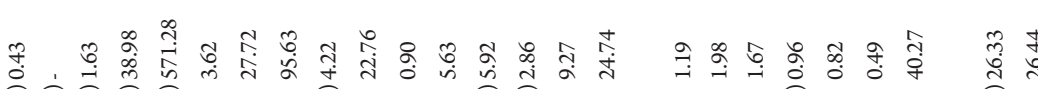

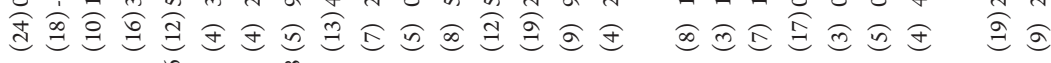

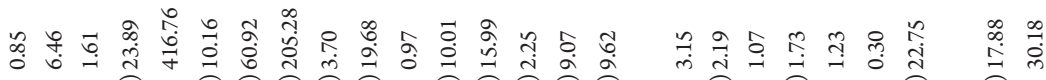

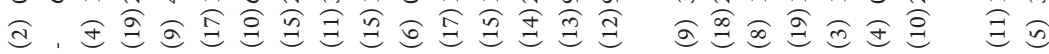

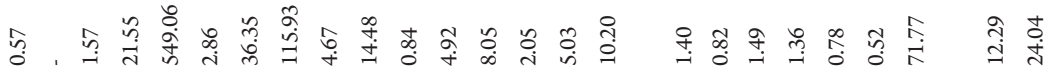

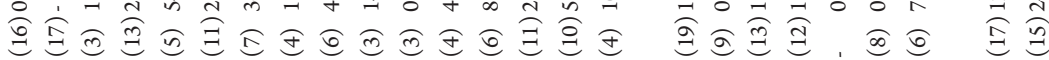

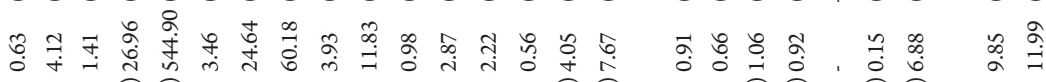

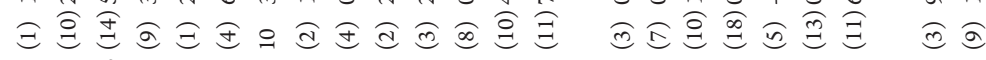

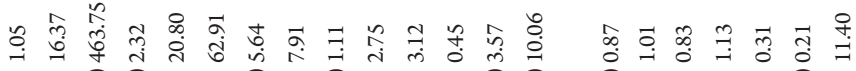

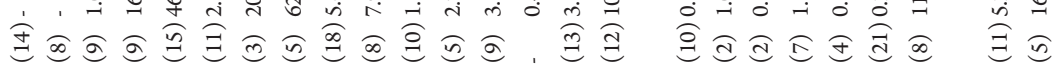

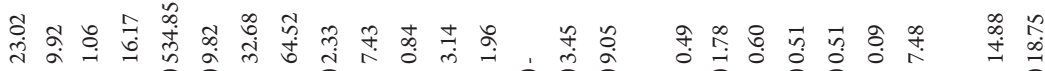

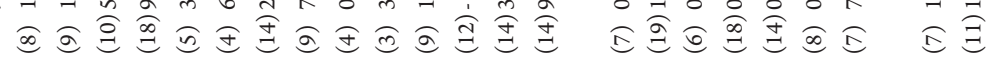

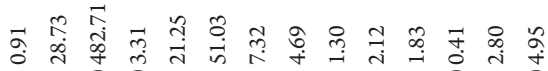

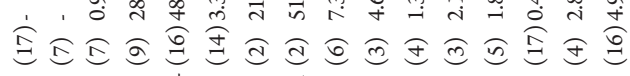

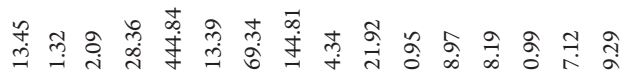

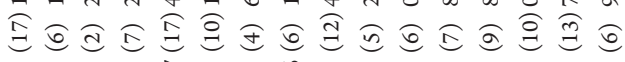

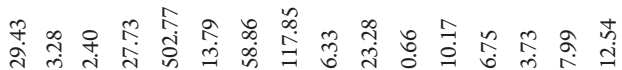

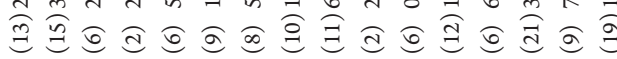

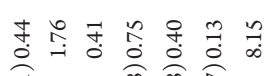
主王王兽兽远远

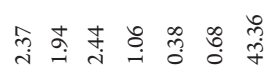
画 $ळ$ 可

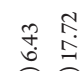
高毫 范 $\sqrt{3} \frac{\sqrt[3]{3}}{3}$

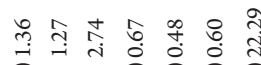

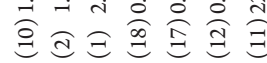

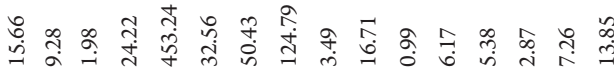

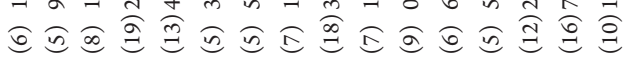

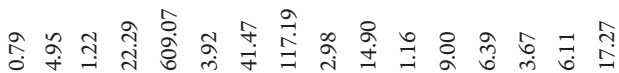
궁

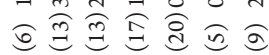

吾

勇家

苟

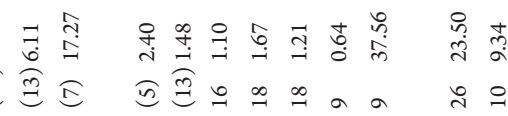

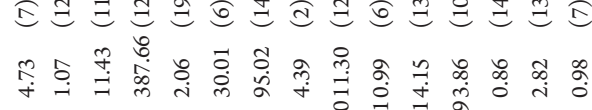

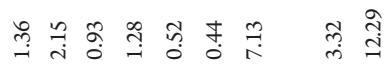
0
0
0
0
0
0
0
0

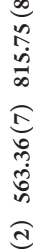

竞 กี่

శึ อ

$\stackrel{\square}{\circ}$

$\stackrel{2}{\Xi}$

잉

$\infty$

守 $, \infty, \infty)$

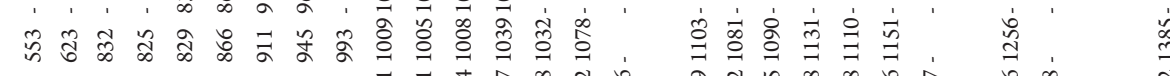

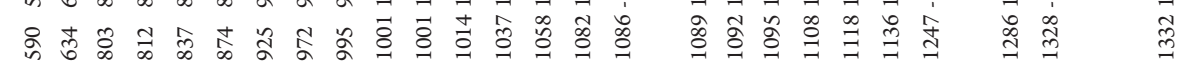

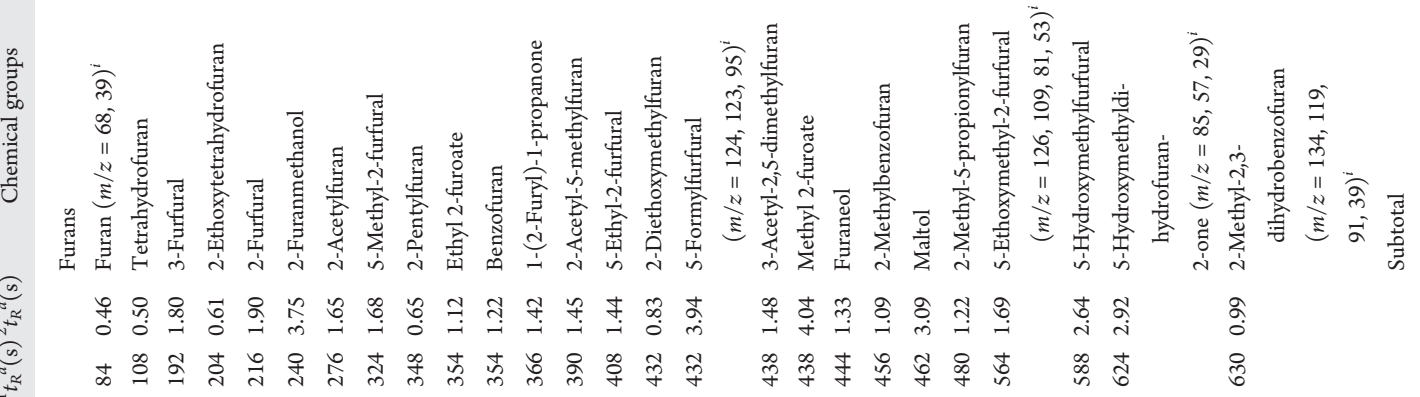

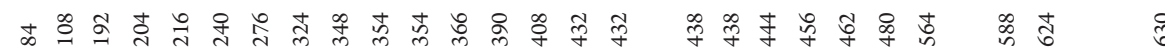




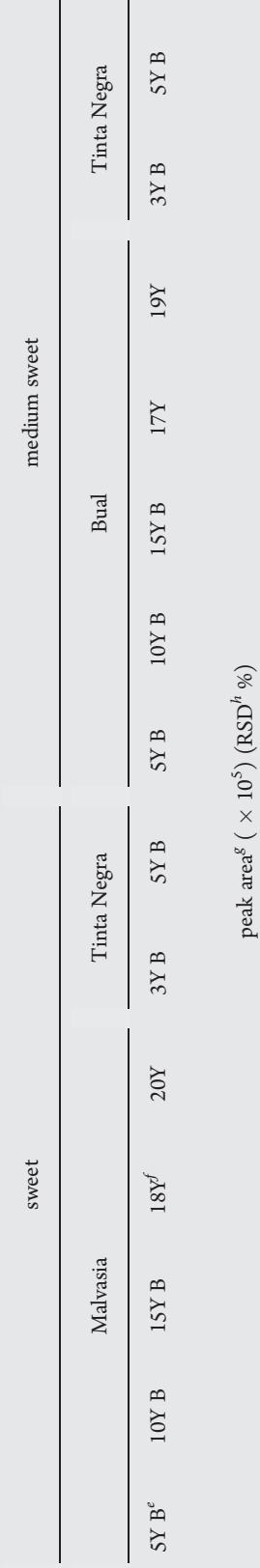

牙

牙苛

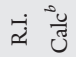

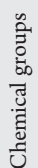

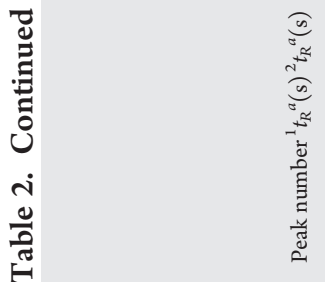

ब오웡

品交离

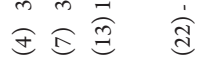

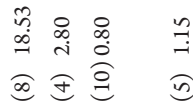

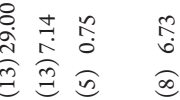

敌字

高远全王苟

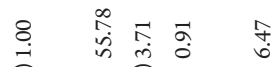

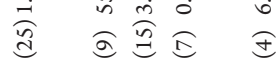

年

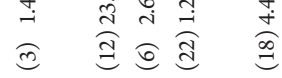

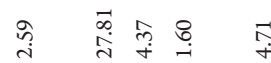

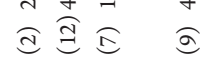

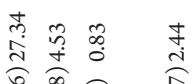

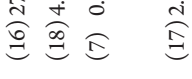

军

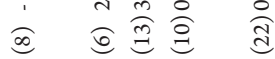

:

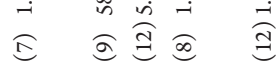

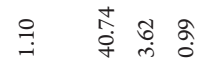

E $E 0$

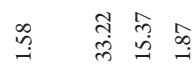

可 0

$\stackrel{\circ}{9}$

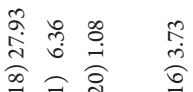

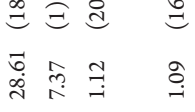

品.

ฌ

ख

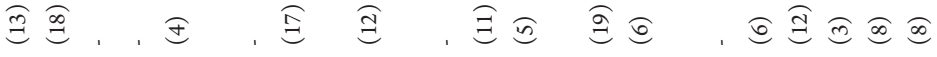

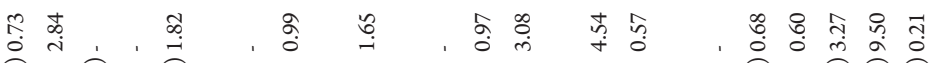

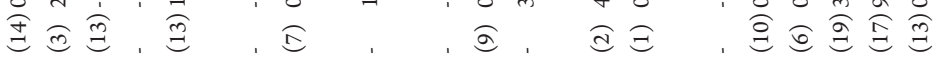

중 8 궁, 章

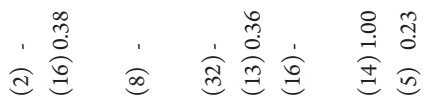

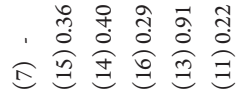

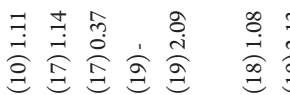

중

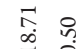

苗

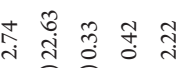

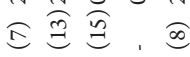

委里

漳

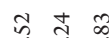

조

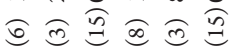

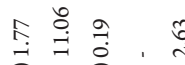

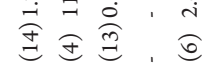

궁

ลี

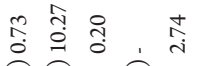

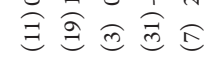

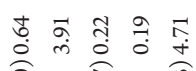

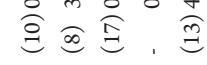

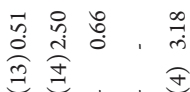

,

亲

远全焉

苛

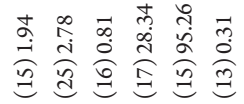

ก̊:

索的

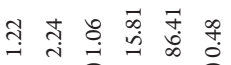

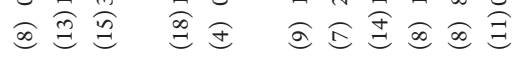

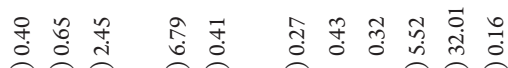

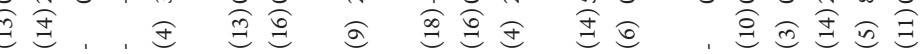

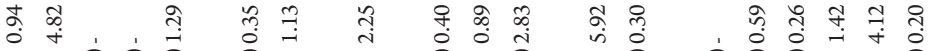

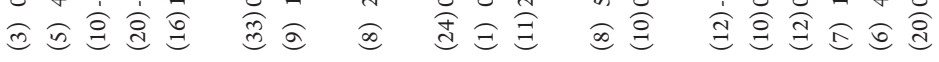

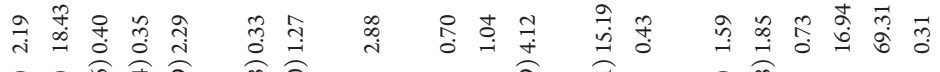

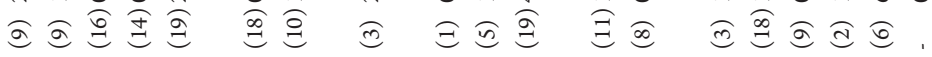

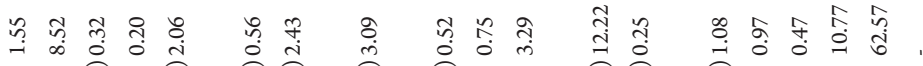

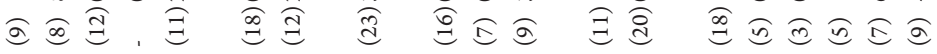

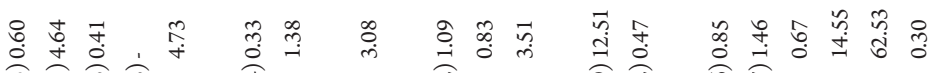

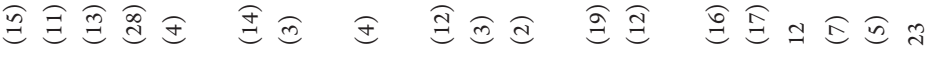

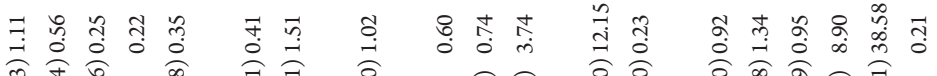

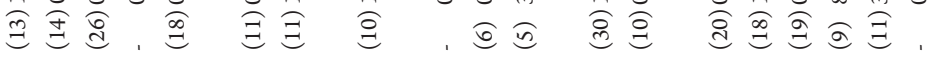

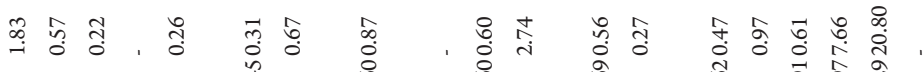

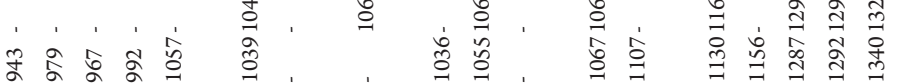

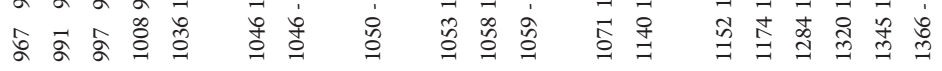

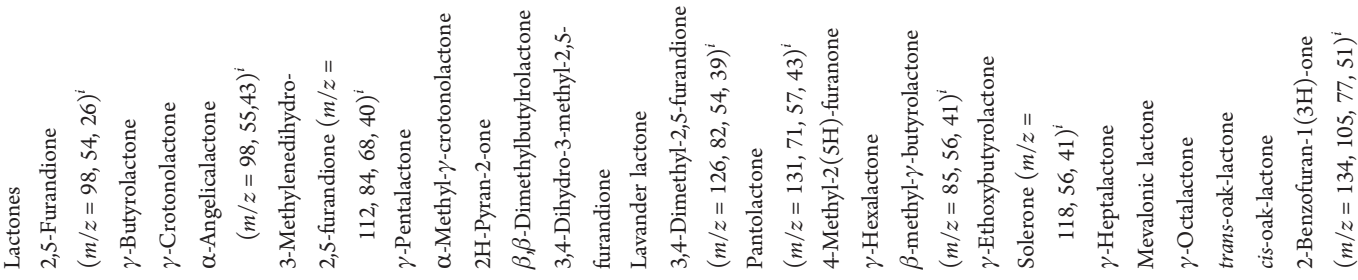

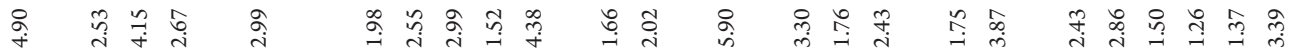

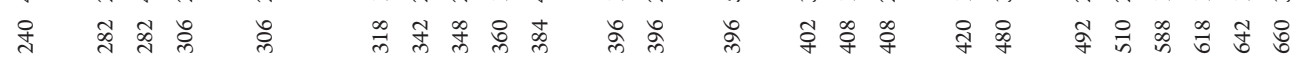

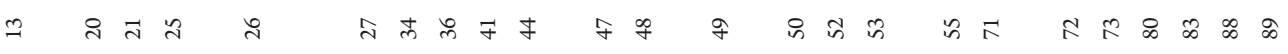




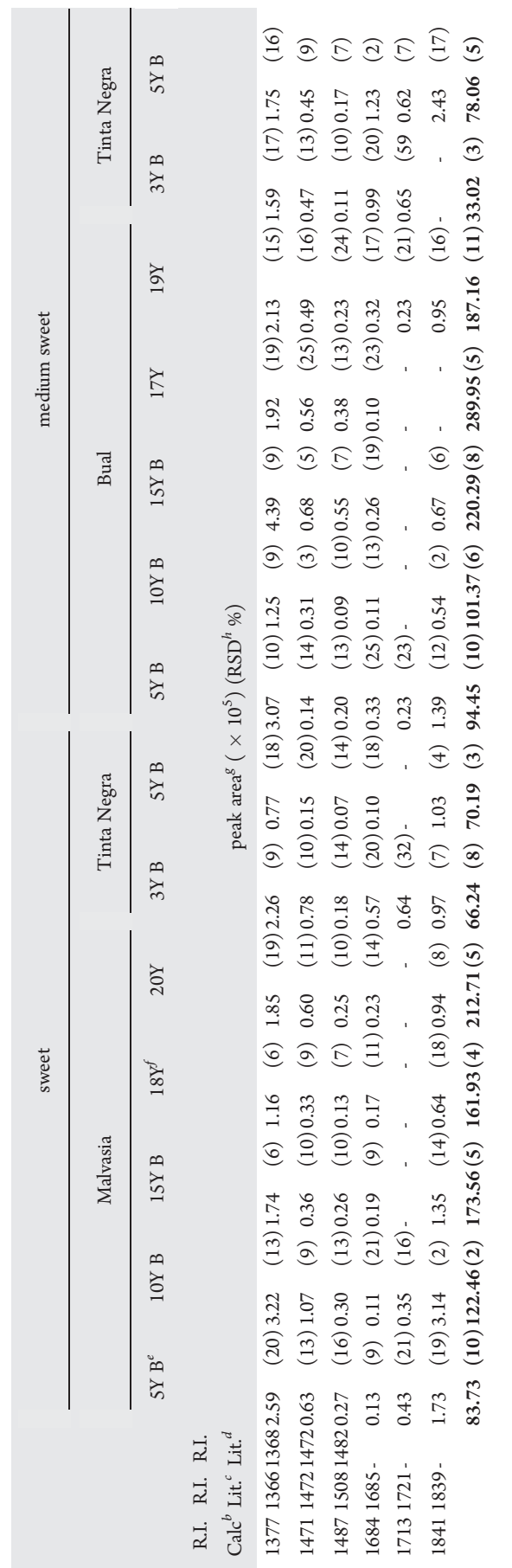

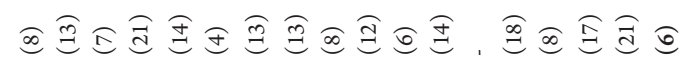

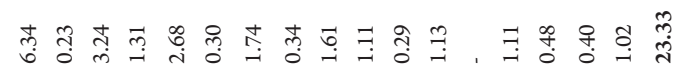

응 $\overline{0}$ 可

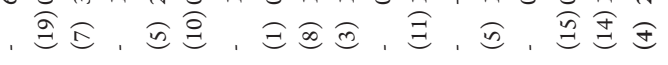

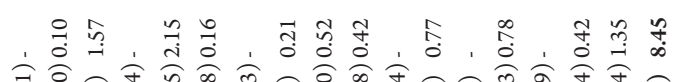

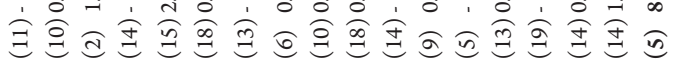

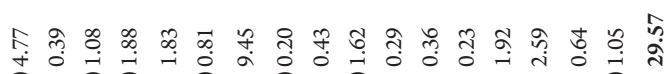

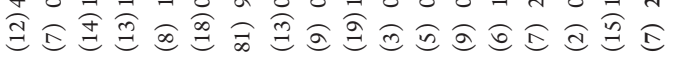

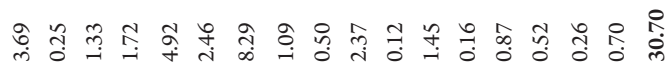

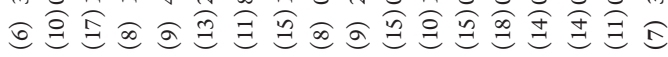

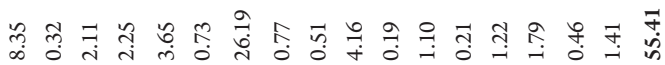

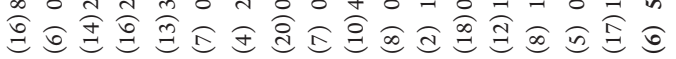

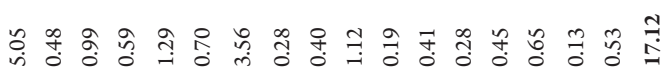

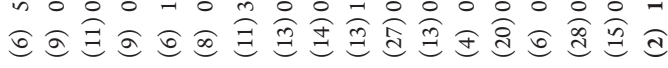

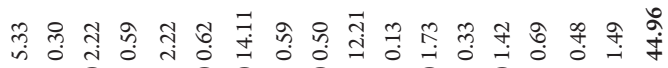

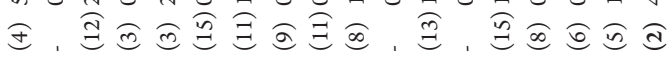

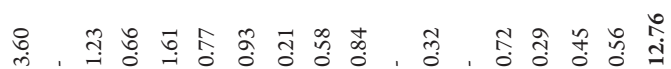

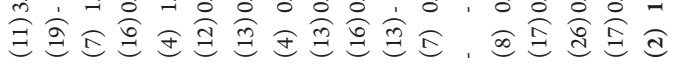

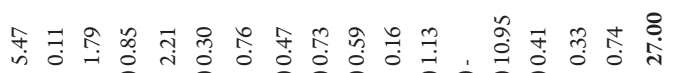

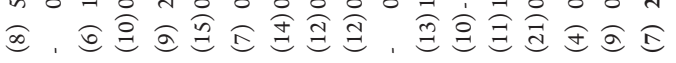

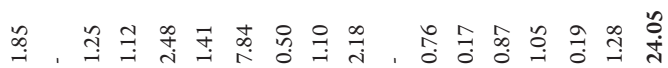

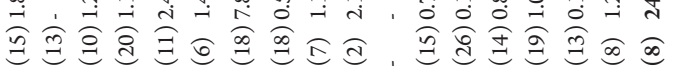

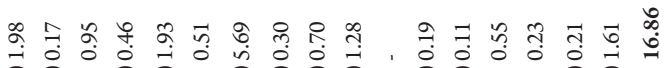

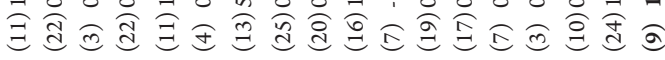

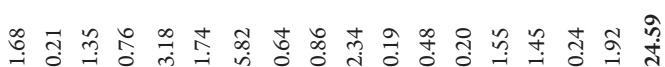

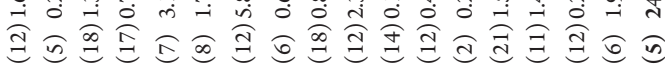

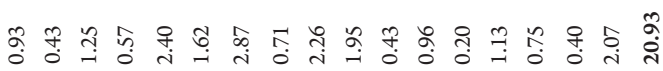

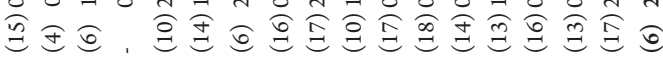

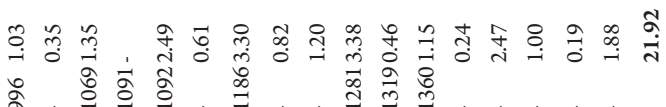

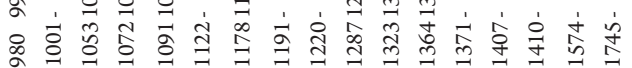

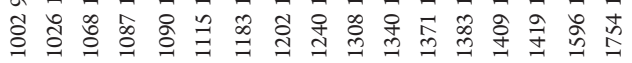

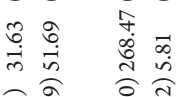

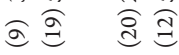

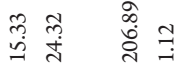
व्वे ब잉 तेळ

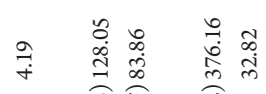
可过 $\widehat{\theta}$

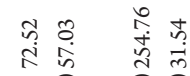
의 $\widehat{\Xi}$ E

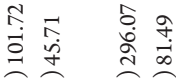

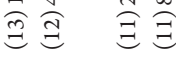

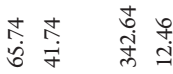
远过是

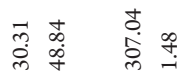

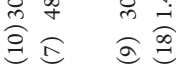

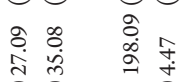
高管 可

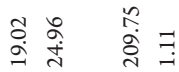

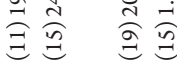

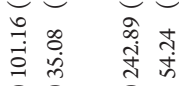
产远

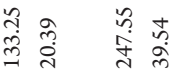

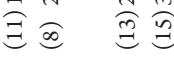

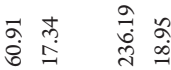
王不 要远

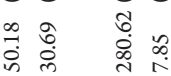

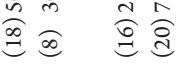

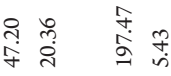

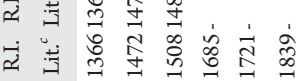

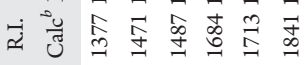

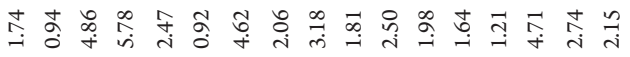

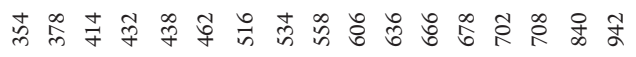




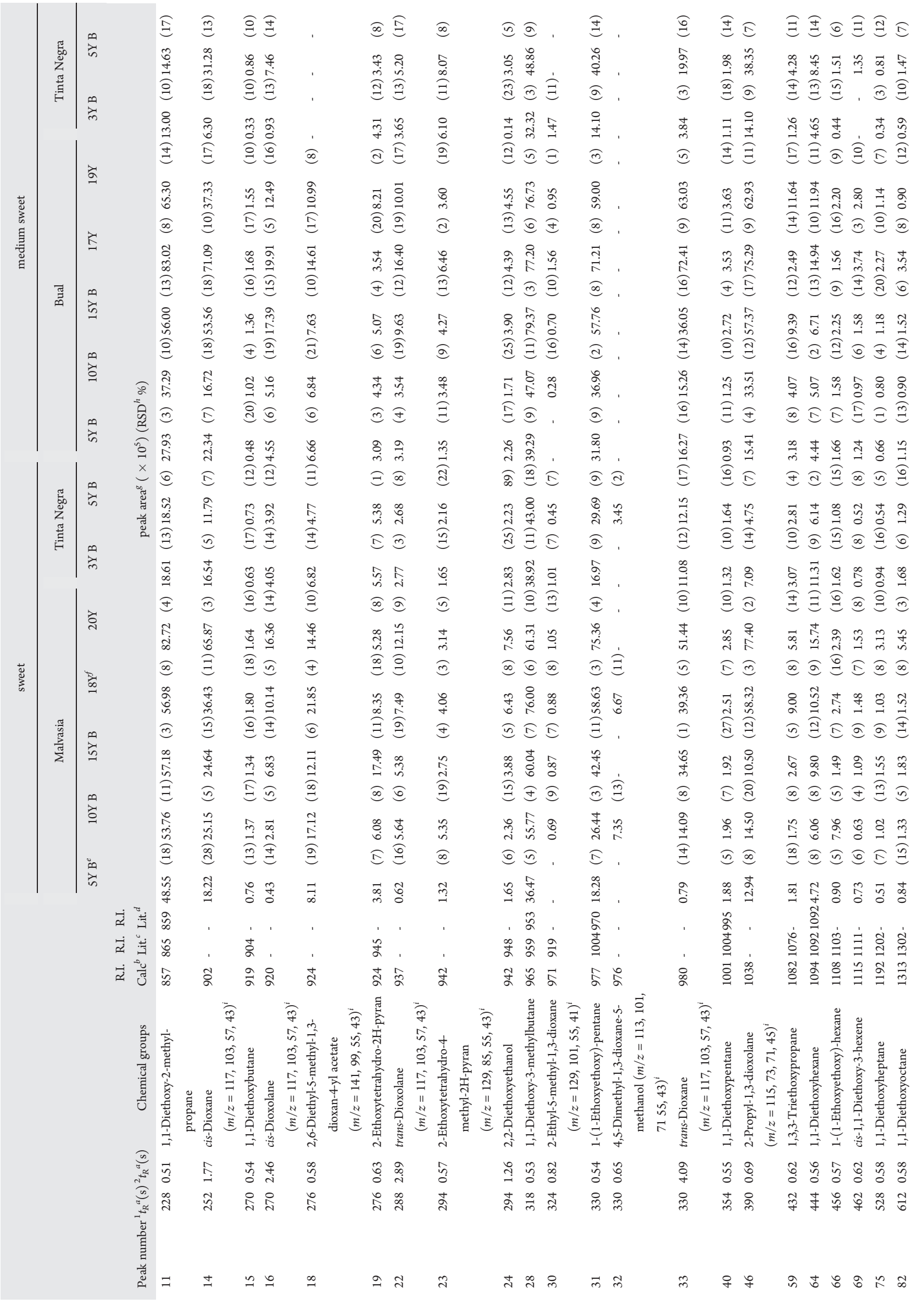




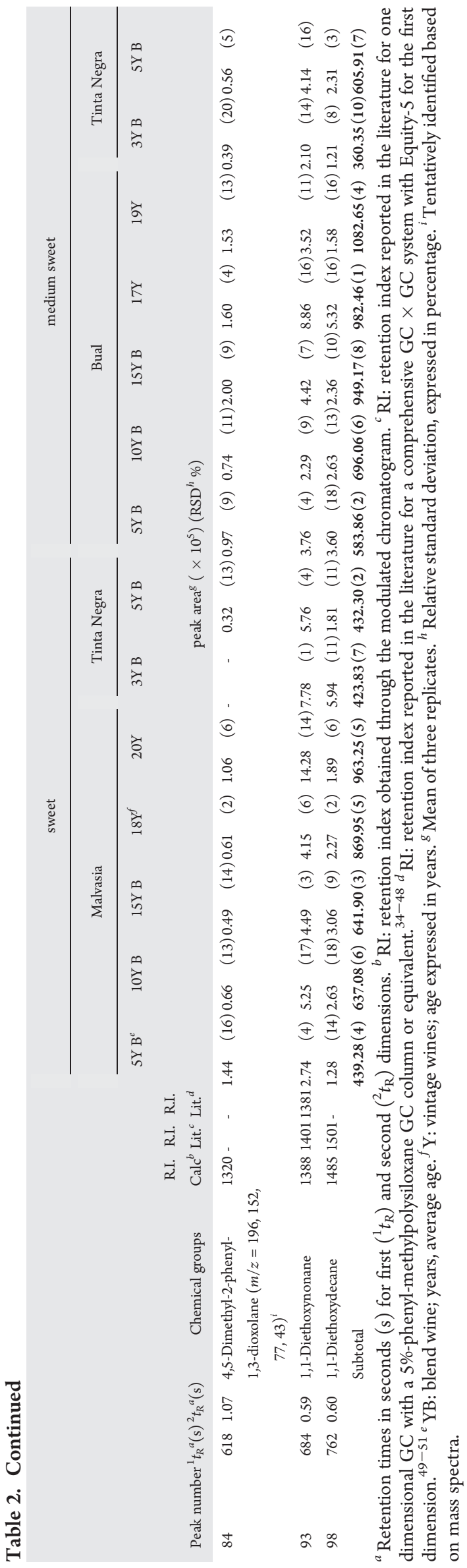

(Figure 2). According to ${ }^{2} \mathrm{D}$ (polarity), it was observed that furan, tetrahydrofuran, alkyl furans (2-ethoxytetrahydrofuran and 2-pentylfuran), and furanic acetal (2-diethoxymethylfuran) exhibited similar polarity. The polarity of these furans increase by the presence of an aromatic ring $\left(-\mathrm{C}_{6} \mathrm{H}_{5}\right)$ (e.g., benzofuran) or a formyl $(-\mathrm{CHO})$ (e.g., furanic aldehydes) group. Concerning furanic aldehydes, the presence of a second - $\mathrm{CHO}$ group in the $\alpha^{\prime}$-position related to the heteroatom of the heterocyclic ring, e. g., 5 -formylfurfural $\left({ }^{1} t_{\mathrm{R}}=432 \mathrm{~s},{ }^{2} t_{\mathrm{R}}=3.94 \mathrm{~s}\right)$ results in a considerable increase in polarity compared to the remaining furanic aldehydes, which were located in the chromatographic space of ${ }^{1} t_{\mathrm{R}}=198-564 \mathrm{~s}$ and ${ }^{2} t_{\mathrm{R}}=1.10-1.90 \mathrm{~s}$ (Figure 2). However, furanic alcohols showed a high polarity compared to furanic aldehydes; consequently, an increase was observed in ${ }^{2} \mathrm{D}$ $\left({ }^{2} t_{\mathrm{R}}=2.64-3.75\right)$. Although, Furaneol $\left({ }^{1} t_{\mathrm{R}}=444 \mathrm{~s},{ }^{2} t_{\mathrm{R}}=1.33 \mathrm{~s}\right)$ is an exception, resultant from the presence of two methyl $\left(-\mathrm{CH}_{3}\right)$ groups in $\alpha$ - and $\alpha^{\prime}$-positions related to the heteroatom, a decreasing in polarity was observed. According to ${ }^{2} \mathrm{D}$, the elution order of furans under study based on their functional groups was alkyl $\sim$ acetal $<$ aromatic ring (benzofuran) $<$ aldehyde < alcohol.

The lactones were organized in lactone/alkyl lactone, anhydride, enolic lactone, and aromatic lactone (Figure 3). The lactone/alkyl lactones are the less polar type; thus, lower ${ }^{2} \mathrm{D}$ values $\left({ }^{2} t_{\mathrm{R}}=0.87-3.30 \mathrm{~s}\right)$ were observed. $\gamma$-Crotonolactone $\left({ }^{1} t_{\mathrm{R}}=282 \mathrm{~s},{ }^{2} t_{\mathrm{R}}=4.15 \mathrm{~s}\right)$ is an exception; the high ${ }^{2} t_{\mathrm{R}}$ was explained due to the presence of $\pi$-bound in the structure and the absence of the $-\mathrm{CH}_{3}$ group. ${ }^{56}$ The anhydride showed higher polarity $\left({ }^{2} t_{\mathrm{R}}=4.38-4.90 \mathrm{~s}\right)$ when compared to lactone/alkyl lactones due to the presence of $\mathrm{RC}=\mathrm{OR}^{\prime}$ group in the $\alpha^{\prime}$ position instead of $\mathrm{H}$. Nevertheless, two exceptions were observed, namely, 3-methylenedihydro-2,5-furandione $\left({ }^{1} t_{\mathrm{R}}=306 \mathrm{~s}\right.$, $\left.{ }^{2} t_{\mathrm{R}}=2.99 \mathrm{~s}\right)$ and 3,4-dimethyl-2,5-furandione $\left({ }^{1} t_{\mathrm{R}}=396 \mathrm{~s},{ }^{2} t_{\mathrm{R}}=\right.$ $2.02 \mathrm{~s}$ ), which demonstrated lower polarity compared to others, due to the a weak $\pi$-bound in the $\beta$-position, and the two $\mathrm{CH}_{3}$ groups in $\beta$ - and $\beta^{\prime}$-positions of heterocyclic ring, respectively. Pantolactone $\left({ }^{1} t_{\mathrm{R}}=396 \mathrm{~s},{ }^{2} t_{\mathrm{R}}=5.90 \mathrm{~s}\right)$, the unique enolic lactone detected, showed the highest polarity of lactones, which may be explained by the presence of the $-\mathrm{OH}$ group in the $\beta$-position of the heterocyclic ring. Thus, according to ${ }^{2} \mathrm{D}$, the elution order of lactones under study based on their functional groups was alkyl $<$ aromatic ring $<$ ketone (anhydride) < alcohol.

The volatile phenols were organized in phenolic ether, alkyloxy phenol, phenol/alkyl phenol, phenolic aldehyde, phenolic ester, and phenoxy alcohol (Figure 4). According to ${ }^{2} \mathrm{D}$, the phenolic ether exhibited the lowest polarity $\left({ }^{2} t_{R}=0.92-1.21 \mathrm{~s}\right)$ compared to alkyloxy phenol, phenol/alkyl phenol, phenolic aldehyde, phenolic ester, and phenoxy alcohol. The presence of the $-\mathrm{OH}$ group in these former compounds increases their polarity; consequently, higher ${ }^{2} t_{R}$ values were observed. Intermediate polarity was observed for alkyloxy phenol $\left({ }^{2} t_{\mathrm{R}}=1.64-\right.$ $2.50 \mathrm{~s}$ ), followed by phenolic ester (e.g., ethyl vanillate, ${ }^{2} t_{\mathrm{R}}=$ $2.74 \mathrm{~s}$ ), phenoxy alcohol (e.g., 2-phenoxyethanol, ${ }^{2} t_{\mathrm{R}}=3.18 \mathrm{~s}$ ), and phenolic aldehyde (e.g., vanillin, ${ }^{2} t_{\mathrm{R}}=4.71 \mathrm{~s}$ ). The alkyl phenols, namely, $o$-cresol, $p$-cresol, and $p$-ethylphenol showed the highest polarity $\left({ }^{2} t_{\mathrm{R}}=4.62-5.78 \mathrm{~s}\right)$, which may be explained by the absence of an $-\mathrm{OCH}_{3}$ group in the $\alpha$-position compared to all other phenols studied. Thus, according to ${ }^{2} \mathrm{D}$, the elution order of volatile phenols under study based on their functional groups was ether (phenolic ether < alkyloxy) < ester < aldehyde $<$ alcohol (alkyl phenol). 


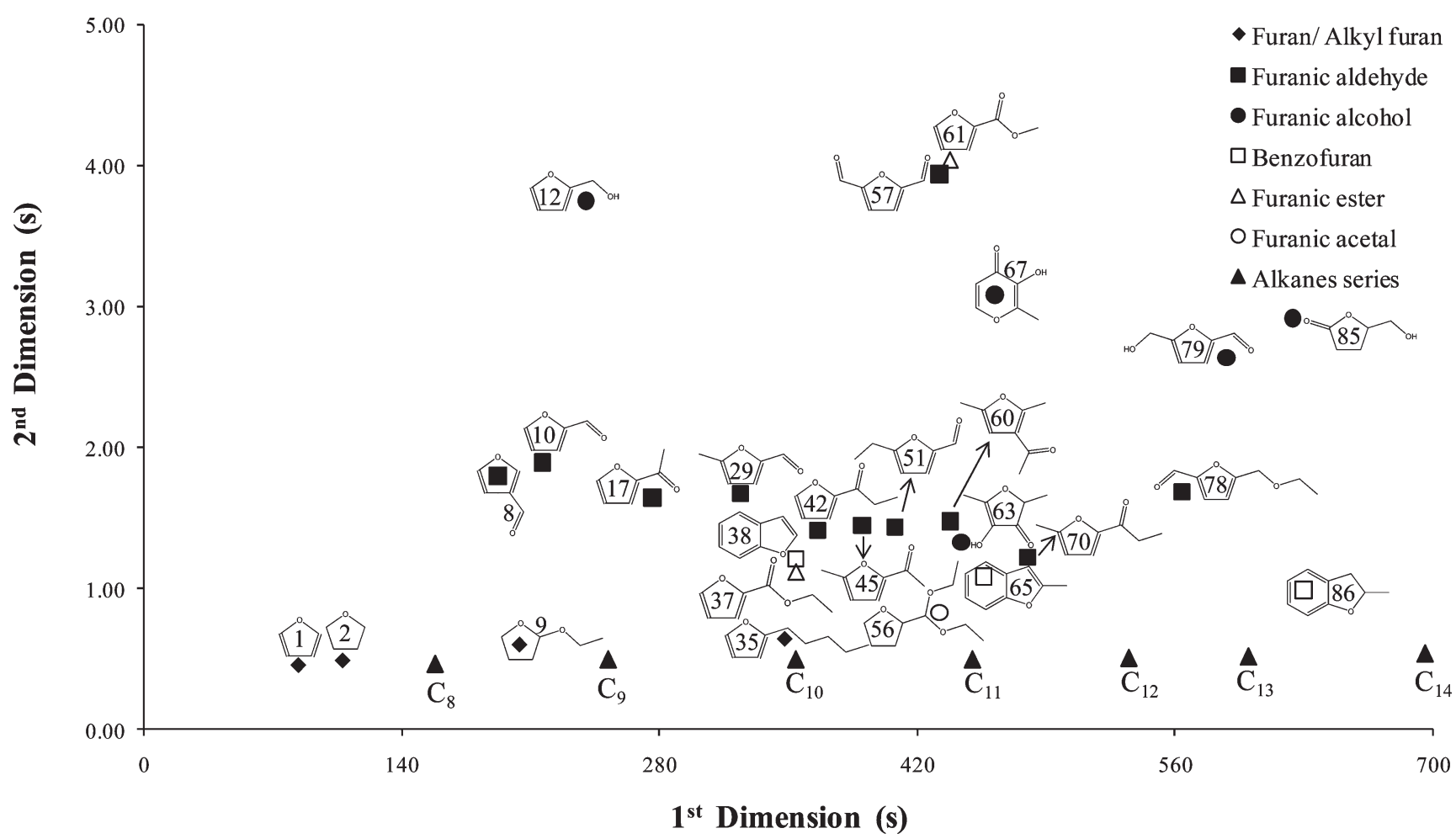

Figure 2. Peak apex plots of furans identified in Madeira wine (attribution of the peak number is shown in Tables 1 and 2).

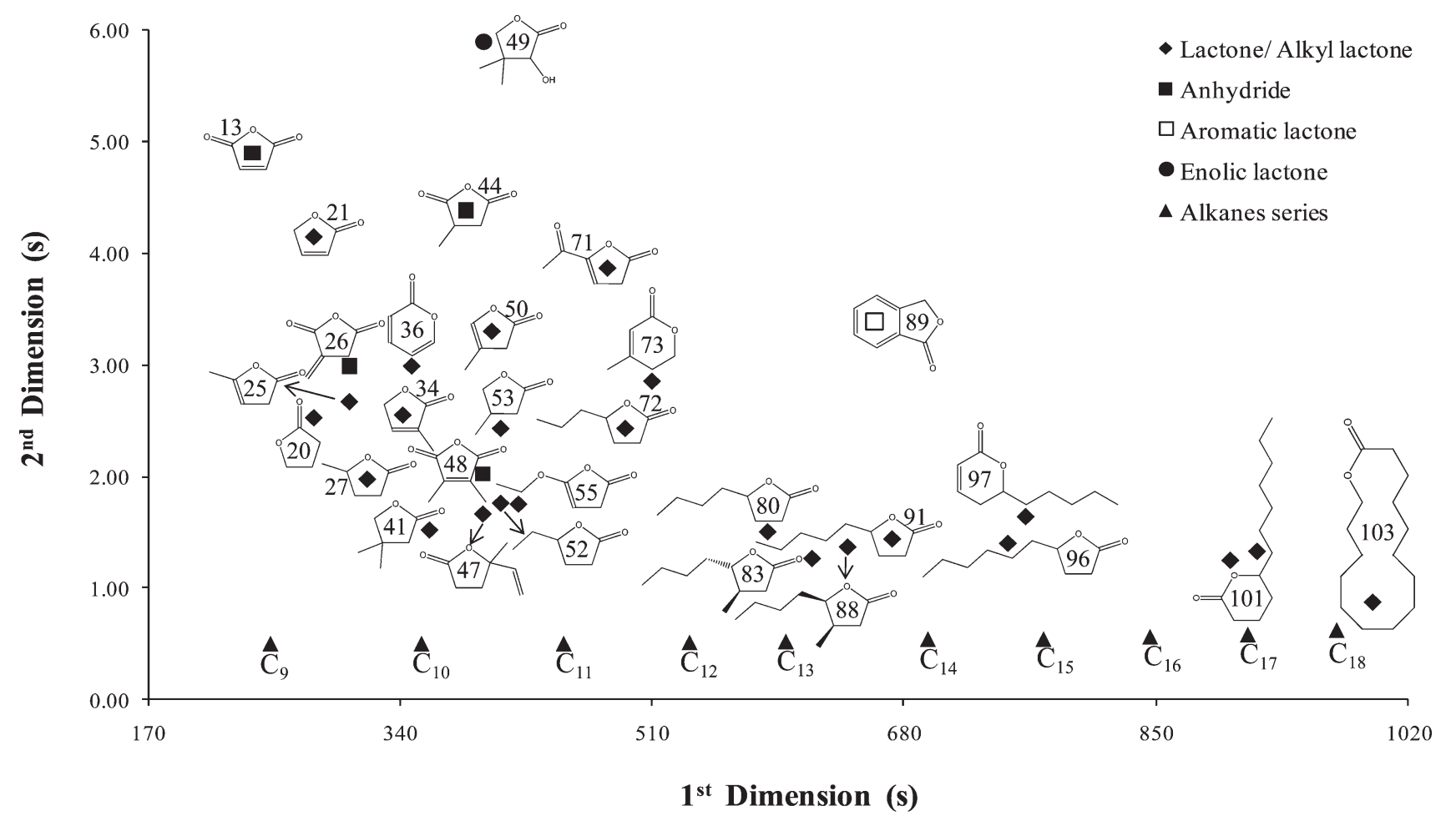

Figure 3. Peak apex plots of lactones identified in Madeira wine (attribution of the peak number is shown in Tables 1 and 2).

Concerning the acetals, three types of structures were observed: alkyl/heterocyclic acetal, alkyl/heterocyclic acetal alcohol, and aromatic acetal. Previous studies only allowed the detection of heterocyclic acetal alcohols. ${ }^{4,10,11,20}$ The alkyl/ heterocyclic acetals are the less polar compounds; afterward, they are the first compounds eluted according ${ }^{2} \mathrm{D}$. For these compounds, the maximum ${ }^{2} t_{\mathrm{R}}$ achieved was $0.82 \mathrm{~s}$. 2-Diethoxyethanol (alkyl acetal alcohol, ${ }^{1} t_{\mathrm{R}}=294 \mathrm{~s},{ }^{2} t_{\mathrm{R}}=1.26 \mathrm{~s}$ ) and 4,5dimethyl-2-phenyl-1,3-dioxolane (aromatic acetal, ${ }^{1} t_{\mathrm{R}}=618 \mathrm{~s}$, ${ }^{2} t_{\mathrm{R}}=1.07 \mathrm{~s}$ ) showed an intermediate polarity due to the presence 


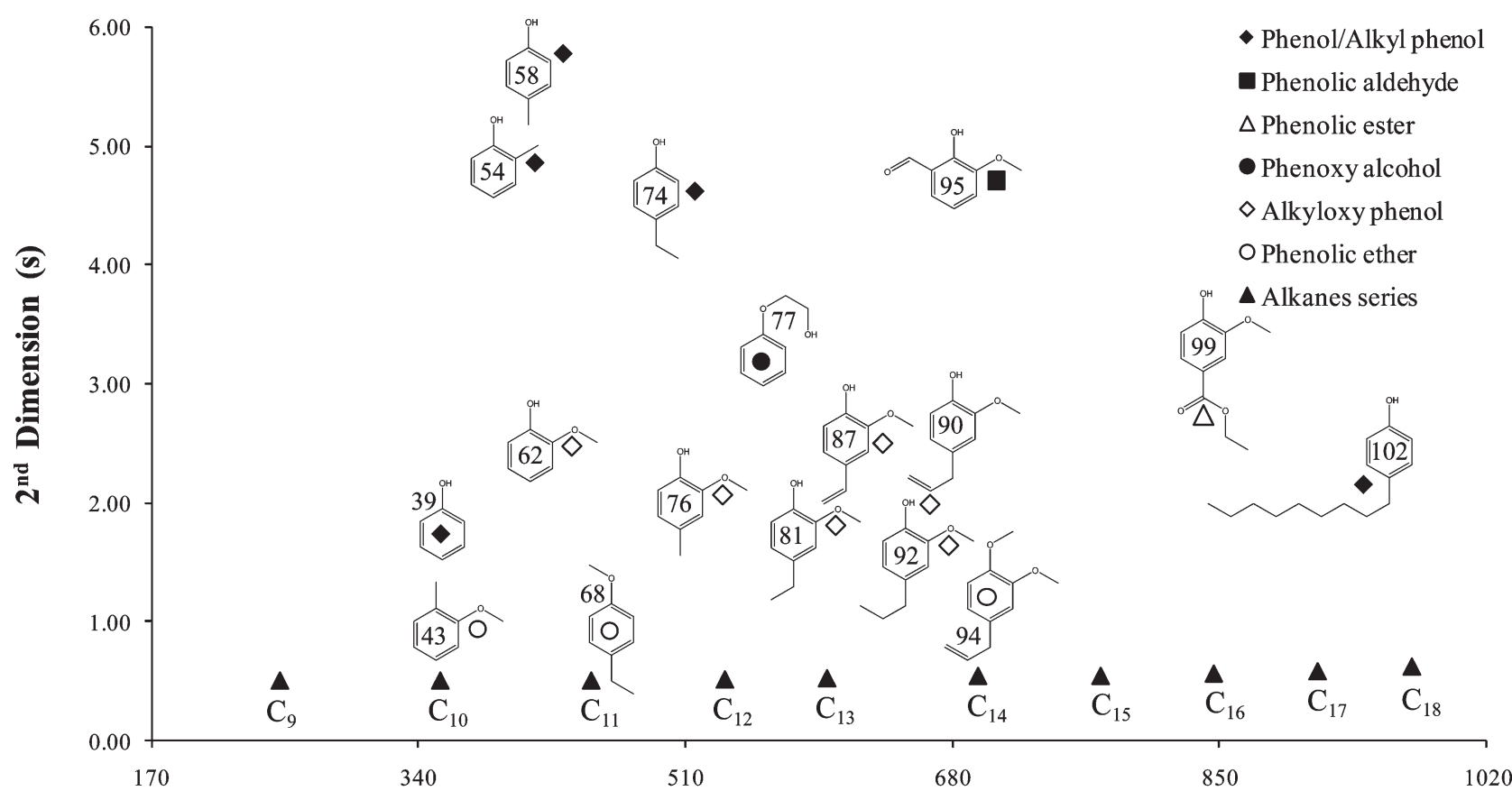

$1^{\text {st }}$ Dimension (s)

Figure 4. Peak apex plots of volatile phenols identified in Madeira wine (attribution of the peak number is shown in Tables 1 and 2).

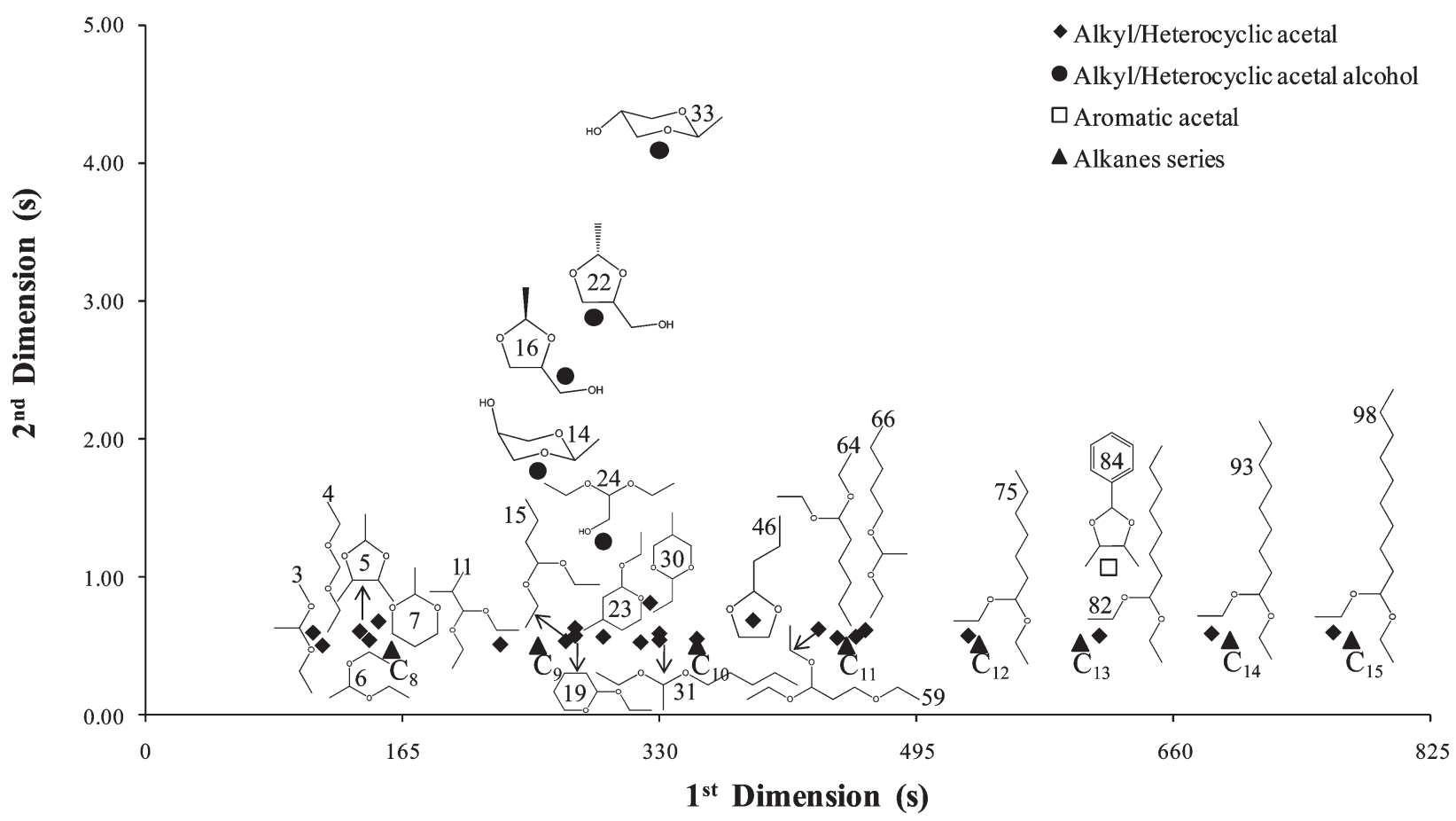

Figure 5. Peak apex plots of acetals identified in Madeira wine (attribution of the peak number shown is in Tables 1 and 2).

of the $-\mathrm{OH}$ and $-\mathrm{C}_{6} \mathrm{H}_{5}$ groups. Otherwise, the four heterocyclic acetal alcohols located in the chromatographic space of ${ }^{1} t_{\mathrm{R}}=252-330 \mathrm{~s}$ and ${ }^{2} t_{\mathrm{R}}=1.77-4.09 \mathrm{~s}$ showed the highest polarity compared to heterocyclic acetals due to the $-\mathrm{OH}$ group in their structure. Thus, according to ${ }^{2} \mathrm{D}$, the elution order of acetals under study was alkyl $<$ aromatic ring $<$ alcohol. Regarding the peak apex of the acetals displayed in Figure 5, it is possible to observe the advantage of the second dimension for the analysis of different acetals.

The structured 2D chromatographic profile arising from ${ }^{1} \mathrm{D}$ volatility and ${ }^{2} \mathrm{D}$ polarity was observed within each chemical group based on the properties and positions of their functional groups, which allow more reliable identifications. Globally, based on the functional group of the chemical families under study, the 
${ }^{2} t_{\mathrm{R}}$ values increase in this way: alkyl $<$ ether $<$ ester $<$ aromatic ring $<$ ketones $\sim$ aldehydes $<$ alcohol. This information is especially useful for classifying unidentified compounds.

Establishment of Potential Age Markers. The GC peak area and RSD (relative standard deviation) values of furans, lactones, volatile phenols, and acetals obtained using HS-SPME/GC $\times$ GC-ToFMS methodology are listed in Table 1 for dry and medium dry, and in Table 2 for sweet and medium sweet Madeira wines.

Furans. The furanic aldehydes, such as 2-furfural and 5methyl-2-furfural, were the predominant furans tentatively identified in the Madeira wines under study. Other furans were also tentatively identified, namely, 2-ethoxytetrahydrofuran, 2-acetylfuran, ethyl 2-furoate, 5-formylfurfural, 5-ethoxymethyl2-furfural, 5-hydroxymethylfurfural, and 5-hydroxymethyldihydrofuran-2-one. Some of these furans have been reported as age markers of Madeira wine, namely, ethyl 2-furoate, 5-ethoxymethyl-2-furfural, and 5-hydroxymethylfurfural, and a similar trend during aging was observed. ${ }^{4,12}$ From the total of 26 furans tentatively identified, only 8 compounds were previously identified in Madeira wines (see Table 1), and as far we know, the 18 furans listed in Tables 1 and 2 are detected for the first time in Madeira wine. In order to evaluate the trend of furans, lactones, volatile phenols, and acetals with the aging process, correlations between GC peak area and age were computed. This approach was only applied to Malvasia and Bual Madeira wines, as they are the unique varieties under study that presented samples with 5 different ages. The other varieties under study only have wines with 2 different ages. A correlation $(r=0.87)$ between total GC peak area of furans and age was found for Bual, while a lower correlation $(r=0.56)$ was achieved for Malvasia, which means that, for Bual, the total GC peak area of furans was $76 \%$ related with age (expressed as $r^{2}$ (coefficient of determination)), whereas for Malvasia, it was only $31 \%$. Furthermore, from 26 furans tentatively identified, only 2 are the highest and positively correlated with wine age, namely, 3-furfural $(r=0.88$ and 0.78 , respectively for Malvasia and Bual), and ethyl 2-furoate ( $r=$ 0.96 and 0.99). Thus, these compounds may be suggested as potential age markers for Malvasia and Bual varieties.

Lactones. $\gamma$-Butyrolactone and cis-oak-lactone were the main lactones detected. Regarding the two isomers of whisky lactones (cis- and trans-oak-lactones), the cis isomer showed the highest GC peak area than trans-isomer. As previously reported, in the wine acidic medium, an easier extraction of cis-oak-lactones occurs. ${ }^{16} \gamma$-Lactones are among the most important compounds from a sensory point of view, and their content tends to increase during the aging process in oak casks (Tables 1 and 2). Similar trends of $\gamma$-lactones during aging were achieved for Madeira and red wines by Câmara et al. ${ }^{4}$ and Cérdan et al., ${ }^{5}$ respectively. From the total of 30 lactones tentatively identified, only 9 compounds were previously identified in Madeira wines (see Table 1), and as far we know, the 21 lactones listed in Tables 1 and 2 are detected for the first time in Madeira wine. The total GC peak area of lactones showed a high correlation $(r=0.93)$ with age in Malvasia, whereas for Bual, a slightly lower correlation $(r=$ 0.71 ) was observed. Thus, for Malvasia the total GC peak area of lactones was $87 \%$ related with age, whereas for Bual, it was only $50 \%$. Moreover, from the 30 lactones tentatively identified, only 5 are the highest and positively correlated with age for Malvasia and Bual varieties, namely, pantolactone $(r=0.78$ and 0.77, respectively, for Malvasia and Bual), $\gamma$-ethoxybutyrolactone ( $r=0.76$ and 0.92), $\gamma$-heptalactone $(r=0.87$ and 0.72$)$, trans-oaklactone $(r=0.83$ and 0.77$)$, and cis-oak-lactone ( $r=0.93$ and 0.82$)$.
Volatile Phenols. The predominant volatile phenols detected were $o$-guaiacol, $p$-ethylphenol, and $p$-ethylguaiacol. During aging, for wines obtained from the Sercial grape variety (alcoholic degree of $16.8 \%^{4}$ ) these compounds increase more remarkably, whereas for Bual wines $\left(17.8 \%{ }^{4}\right)$, a considerable decrease was observed. According to Dias et al., ${ }^{57}$ the content of $p$-ethylphenol and $p$-ethylguaiacol is more accentuated in wines with lower alcoholic degree because the high content reduces the microbial activity of yeast, making the synthesis of ethylphenols difficult. From the total of 17 volatile phenols tentatively identified, 11 compounds were previously identified in Madeira wines (see Table 1), and as far we know, the 6 volatile phenols listed in Tables 1 and 2 are detected for the first time in Madeira wine. Conversely, volatile phenols did not show a linear trend with age for Malvasia $(r=0.05)$ and Bual $(r=0.05)$ varieties. In addition, the individual analysis of all volatile phenols revealed that the GC peak area of these components were not correlated with wine age, with the exception of $p$-cresol $(r=0.79$ and 0.75 , respectively for Malvasia and Bual). This behavior may be explained as the volatile phenols present several origins, i.e., oak, microbiological activity (e.g., Brettanomyces and Dekkara), and hydroxycinnamic acids of wine, ${ }^{23}$ among others.

Acetals. The major acetals detected were diethoxymethane, 1,1diethoxyethane, 1,1-diethoxy-2-methyl-propane, 1,1-diethoxy-3methylbutane, and 1-(1-ethoxyethoxy)-pentane. From the total of 30 acetals tentatively identified, only 4 compounds were previously identified in Madeira wines (see Table 1), and as far we know, the 26 acetals listed in Tables 1 and 2 are detected for the first time in Madeira wine. The white varieties (Malvasia, Bual, Sercial, and Verdelho) presented high total GC peak area of acetals than the red one (Tinta Negra). Similar results were achieved by Cutzach et al., ${ }^{58}$ as the high content of polyphenols in red varieties slowed the oxidation reaction and combined easily with acetaldehyde. For acetals, high correlation with age was observed for Malvasia $(r=0.95)$, and Bual $(r=0.95)$. Similar correlation was observed by Câmara et al. ${ }^{40}$ for heterocyclic acetals alcohols previously identified in Madeira wines. Additionally, other acetals such as 1,1-diethoxy-2-methyl-propane ( $r=0.81$ and 0.86 , respectively, for Malvasia and Bual), 2,2-diethoxyethanol ( $r=0.96$ and $0.84), 1$-(1-ethoxyethoxy)-pentane ( $r=0.96$ and 0.81$), 1,1$ diethoxypentane $(r=0.81$ and 0.94), 2-propyl-1,3-dioxolane $(r=0.86$ and 0.87$)$, and 1,1-diethoxyhexane $(r=0.95$ and 0.83$)$ showed high correlation with age for Malvasia and Bual varieties.

In sum, total GC peak area of acetals showed the highest and positive relationship $(r \geq 0.95)$ with age, whereas the volatile phenols showed the lowest one $(r \leq 0.05)$. Furthermore, from the 103 volatiles tentatively identified to which belong furans, lactones, volatile phenols and acetals, 3-furfural, ethyl 2-furoate, pantolactone, $\gamma$-ethoxybutyrolactone, $\gamma$-heptalactone, trans-oaklactone, and cis-oak-lactone, $p$-cresol, trans-dioxane, trans-dioxolane, 1,1-diethoxy-2-methyl-propane, 2,2-diethoxyethanol, 1-(1-ethoxyethoxy)-pentane, 1,1-diethoxypentane, 2-propyl1,3-dioxolane, and 1,1-diethoxyhexane exhibited the highest and positive correlations $(0.71<r<0.99)$ with wine age. Thus, these compounds may be suggested as potential age markers of Malvasia and Bual wines. In a second step, PCA was applied to autoscaled GC peak areas of furans, lactones, volatile phenols, and acetals, in order to extend the study of potential age markers to all noble varieties (Malvasia, Bual, Sercial, and Verdelho) and to the major variety (Tinta Negra) used to produce Madeira wine, which includes different types of wines and ages as described below. 


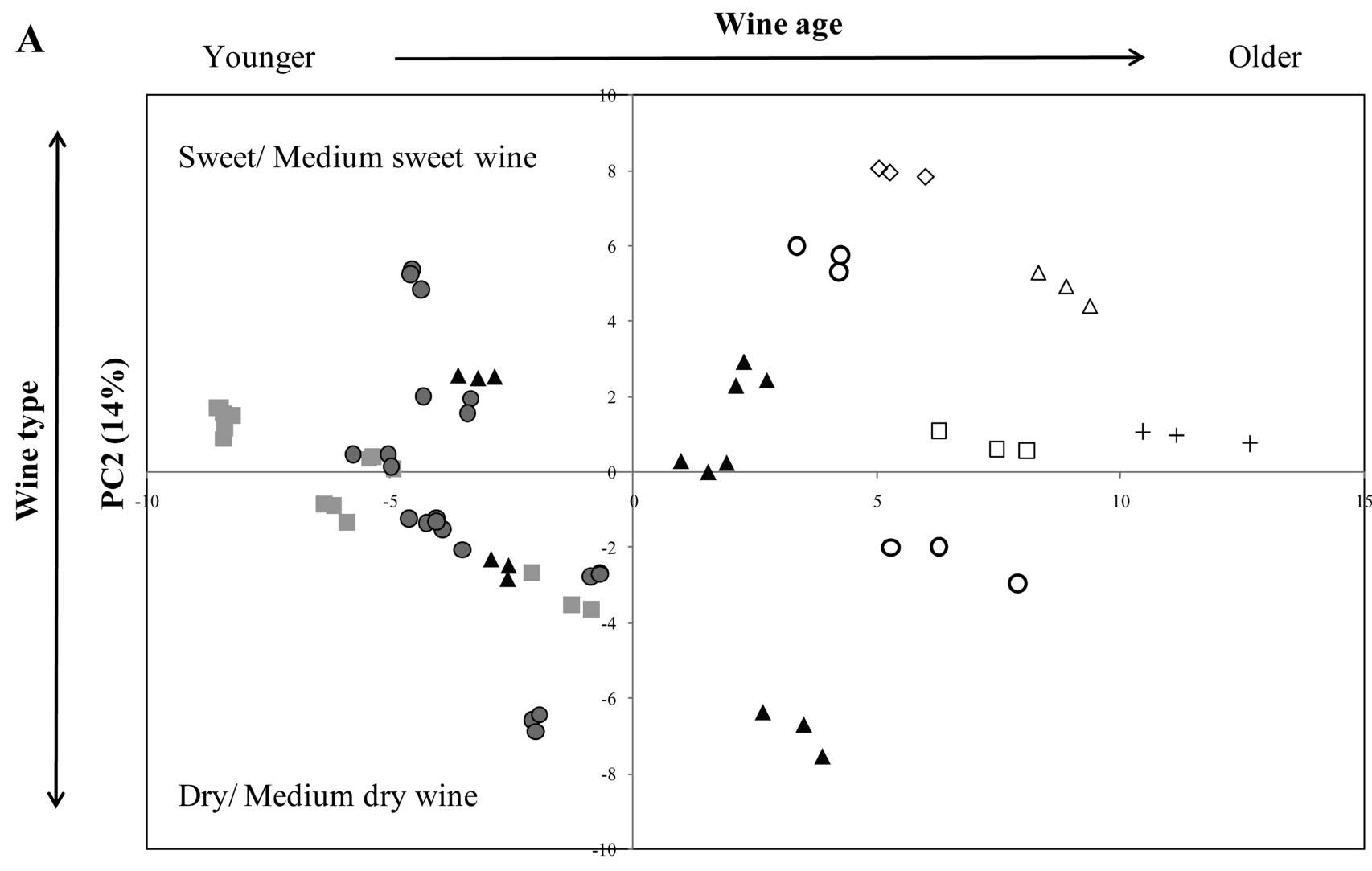

PC1 (32\%)

$\square 3 \mathrm{Y} \circ 5 \mathrm{Y} \Delta 10 \mathrm{Y} \circ 15 \mathrm{Y}+17 \mathrm{Y} \diamond 18 \mathrm{Y} \square 19 \mathrm{Y} \Delta 20 \mathrm{Y}$

B

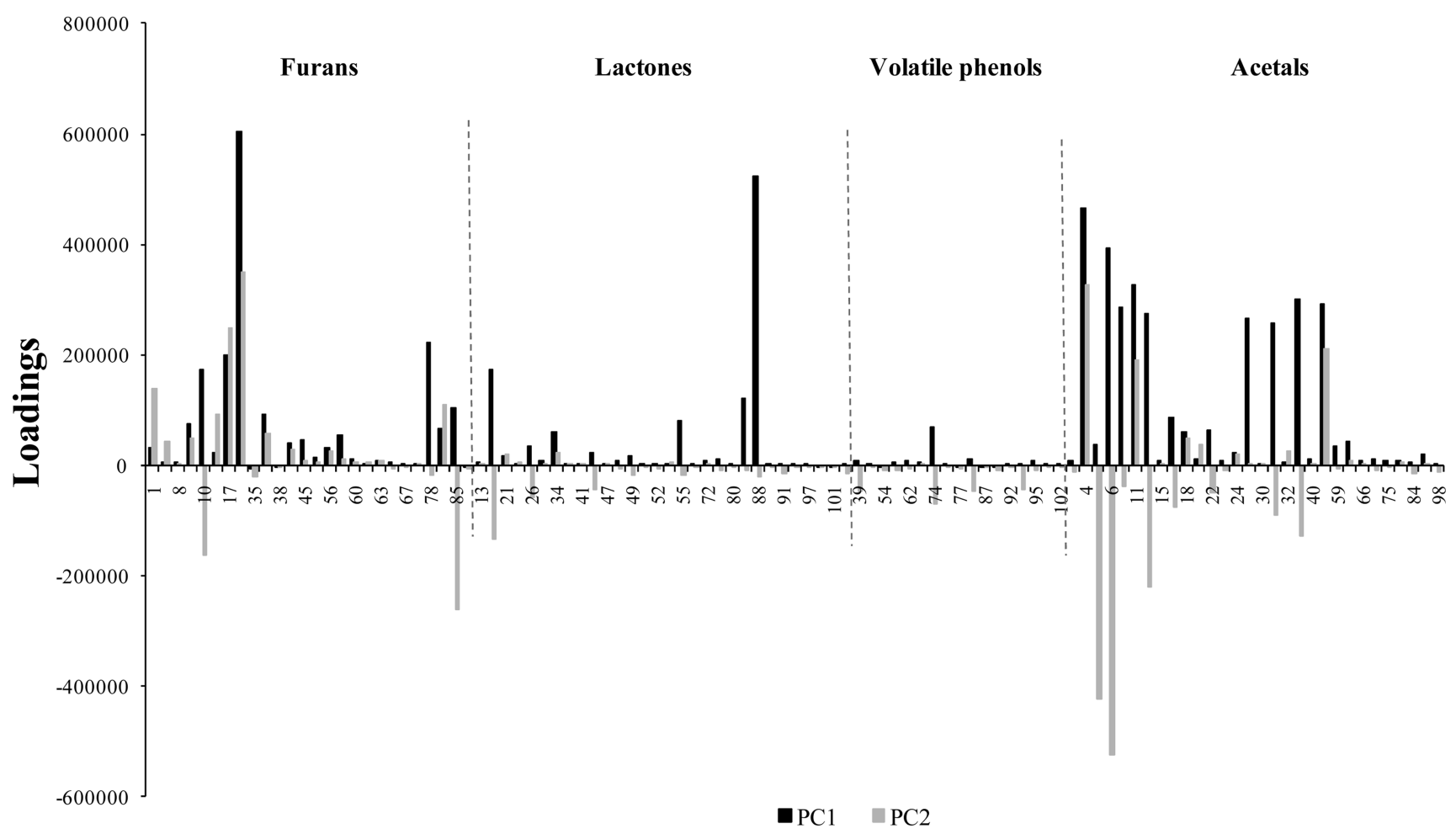

Figure 6. $\mathrm{PC} 1 \times \mathrm{PC} 2$ scores scatter plot (A) and loadings plot profile (B) of GC peak area (attribution of the peak number is shown in Tables 1 and 2). 
Principal Component Analysis. GC peak area of 103 analytical variables (volatile compounds) of the 23 Madeira wines were submitted to a PCA procedure, in order to search for the main sources of variability, to characterize the samples as a function of the detected compounds, and to establish a possible relationship among furans, lactones, volatile phenols and acetals, and wine age. Figure $6 \mathrm{~A}$ shows the score scatter plot of the two first principal components (which explains $46 \%$ of the total variability of the data set), allowing us to organize the Madeira wines by age (as a function of $\mathrm{PC} 1$ axis). Figure $6 \mathrm{~B}$ represents the corresponding loadings plot profile which establishes the relative importance of each volatile for the observed sample distribution of Figure 6A. PC1, which explains $32 \%$ of the total variability, allow one to distinguish Madeira wines as a function of their age. PC2, explaining $14 \%$ of the total variability, shows the organization of the Madeira wines according to their type. With the exception of Tinta Negra, which may be used to produce all types of wines, all other varieties were used to produce a specific type of wine. Sweet (Malvasia) and medium sweet (Bual) Madeira wines (PC2 positive) are characterized by 5-methyl-2-furfural and diethoxymethane, whereas dry (Sercial) and medium dry (Verdelho) were placed in PC2 negative and are described by 1,1-diethoxyethane and 2,4,5trimethyl-1,3-dioxolane. As can be observed in Figure 6A, the Madeira wines could be organized by their type, as well as grape variety according to PC2. According to PC1, the Madeira wines with 3 and $5 \mathrm{Y}$ are projected in PC1 negative, whereas $10 \mathrm{Y}$ is near the origin, except for two Madeira wines, which were located near of $5 \mathrm{Y}$, as these wines correspond to the averaged aging period (blended wines). The Madeira wines with 15, 17, 18,19 , and $20 \mathrm{Y}$ are placed in PC1 positive. Moreover, the Madeira wines could be organized by their style (vintage or blends) according to PC1. The blend Madeira wine is projected in PC1 negative and is near the origin, whereas the vintage is placed in $\mathrm{PC} 1$ positive. Taking into account the loadings plot (Figure 6B), the chemical compounds used as potential age markers were predominantly acetals, namely, diethoxymethane, 1,1-diethoxyethane, 1,1-diethoxy-2-methylpropane, 1-(1-ethoxyethoxy)-pentane, trans-dioxane, and 2-propyl-1,3-dioxolane, and from the other chemical groups studied, 5-methylfurfural (furan) and cis-oak-lactone showed similar contribution to acetals. Finally, it is important to point out that, from these 8 compounds, cis-oak-lactone, transdioxane, 1,1-diethoxy-2-methyl-propane, 1-(1-ethoxyethoxy)pentane, and 2-propyl-1,3-dioxolane were previously (in Establishment of Potential Age Markers) proposed as potential age markers for the Malvasia and Bual varieties. These results suggest that among the chemical groups under study, the acetals are the most important group that could be used as potential age markers of Madeira wines, independently of the variety and the type of wine. Despite the fact that acetals are formed during the fermentation step, its content increases remarkably during the aging process, which may be explained by the oxidative condition that occurred during this step. These oxidative conditions contribute to the increase of aldehyde content, mainly acetaldehyde, and acetals. ${ }^{10}$ Moreover, GC $\times$ GC-ToFMS offered a very useful approach to identify these chemical groups due to the orthogonal systems that reduce coelution and improve the quality of the selection of volatile compounds under study, contributing to the establishment of new potential Madeira wine age markers.

\section{AUTHOR INFORMATION}

\section{Corresponding Author}

*Tel: + 351 234401524. Fax: + 351 234370084. E-mail: smrocha@ua.pt.

\section{Funding Sources}

R. Perestrelo was supported by a Ph.D. grant from Fundação para a Ciência e Tecnologia (SFRH/BD/38855/2007), and we thank the financial support of Research Unit 62/94, QOPNA.

\section{ACKNOWLEDGMENT}

We thank the Madeira Wine Company for kindly providing the samples used in this study, and José Cavaleiro (Full Professor of Organic Chemistry) for the helpful discussions about the nomenclature of organic compounds.

\section{REFERENCES}

(1) Câmara, J. S.; Alves, M. A.; Marques, J. C. Development of headspace solid-phase microextraction-gas chromatography-mass spectrometry methodology for analysis of terpenoids in Madeira wines. Anal. Chim. Acta 2006, 555, 191-200.

(2) Perestrelo, R.; Petronilho, S.; Câmara, J. S.; Rocha, S. M. Comprehensive two-dimensional gas chromatography with time-offlight mass spectrometry combined with solid phase microextraction as a powerful tool for quantification of ethyl carbamate in fortified wines. The case study of Madeira wine. J. Chromatogr., A 2010, 1217, 3441-3445.

(3) Barrera-García, V. D.; Gougeon, R. D.; Voilley, A.; Chassagne, D. Sorption behavior of volatile phenols at the oak wood/wine interface in a model system. J. Agric. Food Chem. 2006, 54, 3982-3989.

(4) Câmara, J. S.; Alves, M. A.; Marques, J. C. Changes in volatile composition of Madeira wines during their oxidative ageing. Anal. Chim. Acta 2006, 563, 188-197.

(5) Cerdán, T. G.; Goñi, D. T.; Azpilicueta, C. A. Accumulation of volatile compounds during ageing of two red wines with different composition. J. Food Eng. 2004, 65, 349-356.

(6) Carrilo, J. D.; Garrido-López, A.; Tena, M. T. Determination of volatile oak compounds in wine by headspace solid-phase microextraction and gas chromatography-mass spectrometry. J. Chromatogr., A 2006, 1102, 25-36.

(7) Simón, B. F.; Estrella Cadahía, E.; Hernández, T.; Estrella, I. Evolution of oak-related volatile compounds in a Spanish red wine during 2 years bottled, after aging in barrels made of Spanish, French and American oak wood. Anal. Chim. Acta 2006, 563, 198-203.

(8) Koussissi, E.; Dourtoglou, V. G.; Ageloussis, G.; Paraskevopoulos, Y.; Dourtoglou, T.; Paterson, A.; Chatzilazarou, A. Influence of toasting of oak chips on red wine maturation from sensory and gas chromatographic headspace analysis. Food Chem. 2009, 114, 1503-1509.

(9) Pérez-Prieto, L. J.; Hera-Orts, M. L.; Lopez-Roca, J. M.; Fernandez-Fernandez, J. I.; Gomez-Plaza, E. Oak-matured wines: Influence of the characteristics of the barrel on wine colour and sensory characteristics. J. Sci. Food Agric. 2003, 83, 1445-1450.

(10) Câmara, J. S.; Marques, J. C.; Alves, A.; Ferreira, A. C. S. Heterocyclic acetals in Madeira wines. Anal. Bioanal. Chem. 2003, 375, 1221-1224.

(11) Pereira, A. C.; Reis, M. S.; Saraiva, P. M.; Marques, J. C. Analysis and assessment of Madeira wine ageing over an extended time period through GC-MS and chemometric analysis. Anal. Chim. Acta 2010, $660,8-21$.

(12) Pereira, A. C.; Reis, M. S.; Saraiva, P. M.; Marques, J. C. Aroma ageing trends in GC/MS profiles of liqueur wines. Anal. Chim. Acta 2010, 659, 93-101.

(13) Martinez, R. G.; Serrana, H. L.; Mir, M. V.; Granados, J. Q.; Martinez, M. C. L. Influence of wood heat treatment, temperature 
and maceration time on vanillin, syringaldehyde, and gallic acid contents in oak wood and wine spirit mixtures. Am. J. Enol. Vitic. 1996, 47, 441-446.

(14) Ortega-Heras, M.; González-Huerta, C.; Herrera, P.; GonzálezSanjosé, M. L. Changes in wine volatile compounds of varietal wines during ageing in wood barrels. Anal. Chim. Acta 2004, 513, 341-350.

(15) Moreno, N. J.; Azpilicueta, C. A. Binding of oak volatile compounds by wine lees during simulation of wine ageing. LWT Food Sci. Technol. 2007, 40, 619-624.

(16) Ortega-Heras, M.; González-Sanjosé, M. L.; González-Huerta, C. Consideration of the influence of aging process, type of wine and oenological classic parameters on the levels of wood volatile compounds present in red wines. Food Chem. 2007, 103, 1434-1448.

(17) Ramírez-Jiménez, A.; Garcia-Villanova, B.; Guerra-Hernández, E. Hydroxymethylfurfural and methylfurfural content of selected bakery products. Food Res. Int. 2000, 33, 833-838.

(18) Silva, H. O.; Pinho, P. G.; Machado, B. P.; Hogg, T.; Marques, J. C.; Câmara, J. S.; Albuquerque, F.; Ferreira, A. C. S. Impact of forcedaging process on Madeira wine flavor. J. Agric. Food Chem. 2008, 56, 11989-11996.

(19) Alañón, M. E.; Rubio, H.; Díaz-Maroto, M. C.; Pérez-Coello, M. S. Monosaccharide anhydrides, new markers of toasted oak wood used for ageing wines and distillates. Food Chem. 2010, 119, 505-512.

(20) Schneider, R.; Baumes, R.; Bayonove, C.; Razungles, A. Volatile compounds involved in the aroma of sweet fortified wines (Vins Doux Naturels) from Grenache Noir. J. Agric. Food Chem. 1998, 46, 3230-3237.

(21) Belitz, H. D.; Grosch, W.; Schieberle, P. Food Chemistry, 3rd ed.; Springer-Verlag: Berlin, Germany, 2004; Chapter 5, p 342.

(22) Guchu, E.; Díaz-Maroto, M. C.; Díaz-Maroto, I.J.; Vila-Lameiro, P.; Pérez-Coello, M. S. Influence of the species and geographical location on volatile composition of spanish oak wood (Quercus petraea Liebl. and Quercus robur L.). J. Agric. Food Chem. 2006, 54, 3062-3066.

(23) Rodríguez-Bencomo, J. J.; Ortega-Heras, M.; Perez-Magariño, S.; González-Huerta, C. Volatile compounds of red wines macerated with Spanish, American, and French oak chips. J. Agric. Food Chem. 2009, 57, 6383-6391.

(24) Schreier, P. Flavor composition of wines: a review. CRC Crit. Rev. Food Sci. Nutr. 1979, 12, 59-67.

(25) Souza, P. O.; Cardeal, Z. L.; Augusti, R.; Morrison, P.; Marriott, P. J. Determination of volatile compounds in Brazilian distilled cachaça by using comprehensive two-dimensional gas chromatography and effects of production pathways. J. Chromatogr., A 2009, $1216,2881-2890$

(26) Górecki, T.; Panić, O.; Oldridge, N. Recent advances in comprehensive two-dimensional gas chromatography $(\mathrm{GC} \times \mathrm{GC})$. J. Liq. Chromatogr. Relat. Technol. 2006, 29, 1077-1104.

(27) Herrero, M.; Ibáñez, E.; Cifuentes, A.; Bernal, J. Multidimensional chromatography in food analysis. J. Chromatogr., A 2009, 1216, 7110-7129.

(28) Zrostlíková, J.; Hajšlová, J.; Čajka, T. Evaluation of twodimensional gas chromatography-time-of-flight mass spectrometry for the determination of multiple pesticide residues in fruit. J. Chromatogr., A 2003, 1019, 173-186.

(29) Tranchida, P. Q.; Dugo, P.; Dugo, G.; Mondello, L. Comprehensive two-dimensional chromatography in food analysis. J. Chromatogr., A 2004, 1054, 3-16.

(30) Song, S. M.; Marriott, P.; Kotsos, A.; Drummer, O. H.; Wynne, P. Comprehensive two-dimensional gas chromatography with time-offlight mass spectrometry (GC x GC-TOFMS) for drug screening and confirmation. Forensic Sci. Int. 2004, 143, 87-101.

(31) Mondello, L.; Tranchida, P. Q.; Dugo, P.; Dugo, G. Comprehensive two-dimensional gas chromatography-mass spectrometry: a review. Mass Spectrom. Rev. 2008, 27, 101-124.

(32) Dallüge, J.; Beens, J.; Brinkman, U. A. T. Comprehensive twodimensional gas chromatography: a powerful and versatile analytical tool. J. Chromatogr., A 2003, 1000, 69-108.
(33) Van Den Dool, H.; Kratz, P. D. A generalization of the retention index system including linear temperature programmed gas-liquid partition chromatography. J. Chromatogr., A 1963, 11, 463-471.

(34) Adams, R. P. Identification of Essential Oil Components by Gas Chromatography/Mass Spectrometry; Allured Publishing Corp.: Carol Stream, IL, 1995.

(35) Aaslyng, M. D.; Elmore, J. S.; Mottram, D. S. Comparison of the aroma characteristics of hydrolyzed-acid and enzyme-hydrolyzed vegetable proteins produced from soy. J. Agric. Food Chem. 1998, 46, 5225-5231.

(36) Ansorena, D.; Astiasarán, I.; Bello, J. Influence of the simultaneous addition of the protease flavourzyme and the lipase novozyme 677BG on dry fermented sausage compounds extracted by SDE and analyzed by GC-MS. J. Agric. Food Chem. 2000, 48, 2395-2400.

(37) Jordan, M. J.; Margaria, C. A.; Shaw, P. E.; Goodner, K. L. Aroma active components in aqueous kiwi fruit essence and kiwi fruit puree by GC-MS and multidimensional GC/GC-O. J. Agric. Food Chem. 2002, 50, 5386-5390.

(38) Whitfield, F. B.; Mottram, D. S. Heterocyclic volatiles formed by heating cysteine or hydrogen sulfide with 4-hydroxy-5-methyl3(2H)-furanone at $\mathrm{pH}$ 6.5. J. Agric. Food Chem. 2001, 49, 816-822.

(39) Marogiu, B.; Piras, A.; Pani, F.; Porcedda, S.; Bailero, M. Extraction, separation and isolation of essential oils from natural matrices by supercritical $\mathrm{CO}_{2}$. Flavour Frag. J. 2003, 18, 505-509.

(40) Leffingwell, J. C.; Alford, E. D. Volatile constituents of perique tobacco. J. Environ. Agric. Food Chem. 2005, 4, 899-915.

(41) Mass Spectra Library NIST 2.0, 2005.

(42) Acree, T. E.; Arn, H. Flavornet; Cornel University, New York, 2010 (http://www.flavornet.org).

(43) Pino, J. A.; Mesa, J.; Munoz, Y.; Marti, M. P.; Marbot, R. Volatile Components from Mango (Mangifera indica L.) Cultivars. J. Agric. Food Chem. 2005, 53, 2213-2223.

(44) D’Arcy, B. R.; Rintoul, G. B.; Rowland, C. Y.; Blackman, A. J. Composition of Australian honey extractives. 1. Norisoprenoids, monoterpenes, and other natural volatiles from blue gum (Eucalyptus leucoxylon) and yellow box (Eucalyptus melliodora) honeys. J. Agri. Food Chem. 1997, 45, 1834-1843.

(45) Fan, W.; Qian, M. C. Characterization of aroma compounds of Chinese "Wuliangye" and "Jiannanchun" liquors by aroma extract dilution analysis. J. Agric. Food Chem. 2006, 54, 2695-2704.

(46) Zimmermann, M.; Schieberle, P. Important odorants of sweet bell pepper powder (Capsicum annuum cv. annuum): differences between samples of Hungarian and Morrocan origin. Eur. Food Res. Technol. 2000, 211, 175-180.

(47) Qian, M.; Reineccius, G. Potent aroma compounds in Parmigiano Reggiano cheese studied using a dynamic headspace (purge-trap) method. Flavour Frag. J. 2003, 18, 252-259.

(48) Zhao, Y.; Li, J.; Xu, Y.; Duan, H.; Fan, W; Zhao, G. Extraction, preparation and identification of volatile compounds in Changyu $\mathrm{XO}$ Brandy. Chinese J. Chromatogr. 2008, 26, 212-222.

(49) Cardeal, Z. I.; Souza, P. P.; Silva, M. D. R. G.; Marriott, P. J. Comprehensive two-dimensional gas chromatography for fingerprint pattern recognition in cachaça production. Talanta 2008, 74, 793-799.

(50) Babushok, V. I.; Zenkevich, I. G. Retention indices for most frequently reported essential oil compounds in GC. Chromatographia 2009, 69, 257-269.

(51) Robinson, A. L.; Boss, P. K.; Heymann, H.; Solomon, P. S.; Trengove, R. D. Development of a sensitive non-targeted method for characterizing the wine volatile profile using headspace solid-phase microextraction comprehensive two-dimensional gas chromatography time-of-flight mass spectrometry. J. Chromatogr., A 2011, 1218, 504-517.

(52) R Development Core Team. R: A Language and Environment for Statistical Computing; R Foundation for Statistical Computing: Vienna, Austria, 2009; http://www.R-project.org.

(53) Jolliffe, I. T. Principal Component Analysis, 2nd ed.; Springer: New York, 2002.

(54) Silva, I.; Rocha, S. M.; Coimbra, M. A.; Marriott, P. J. Headspace solid-phase microextraction combined with comprehensive two-dimensional gas chromatography time-of-flight mass spectrometry for the 
determination of volatile compounds from marine salt. J. Chromatogr., $A$ 2010, 1217, 5511-5521.

(55) Cardeal, Z. I.; Marriott, P. J. Comprehensive two-dimensional gas chromatography-mass spectrometry analysis and comparison of volatile organic compounds in Brazilian cachaça and selected spirits. Food Chem. 2009, 112, 747-755.

(56) Carey, F. A.; Sunderg, R. J. Advanced Organic Chemistry, 5th ed.; Springer-Verlag: Berlin, Germany, 2007; Chapters 1, 8.

(57) Dias, L.; Silva, S. P.; Tavares, M.; Ferreira, M. M.; Loureiro, V. Factors affecting the production of 4-ethylphenol by the yeast Dekkera bruxellensis in enological conditions. Food Microbiol. 2003, 20, 377-384.

(58) Cutzach, I.; Chatonnet, P.; Dubourdieu, D. Study of the formation mechanisms of some volatile compounds during the aging of sweet fortified wines. J. Agric. Food Chem. 1999, 47, 2837-2846.

(59) Perestrelo, R.; Fernandes, A.; Albuquerque, F. F.; Marques, J. C.; Câmara, J. S. Analytical characterization of the aroma of Tinta Negra Mole red wine: Identification of the main odorants compounds. Anal. Chim. Acta 2006, 563, 154-164.

(60) Campo, E.; Ferreira, V.; Escudero, A.; Marqués, J C.; Cacho, J. Quantitative gas chromatography-olfactometry and chemical quantitative study of the aroma of four Madeira wines. Anal. Chim. Acta 2006, $563,180-187$. 